Draft Version OCtOBer 30, 2018

Preprint typeset using $\mathrm{LAT}_{\mathrm{E}} \mathrm{X}$ style emulateapj v. 08/22/09

\title{
GLOBAL MODELS FOR THE EVOLUTION OF EMBEDDED, ACCRETING PROTOSTELLAR DISKS
}

\author{
Kaitlin M. Kratter and Christopher D. Matzner \\ Department of Astronomy and Astrophysics, University of Toronto, Toronto, ON M5S 3H4 \\ Mark R. Krumholz ${ }^{1}$ \\ Department of Astrophysical Sciences, Princeton University, Princeton, NJ 08544-1001 \\ Draft version October 30, 2018
}

\begin{abstract}
Most analytic work to date on protostellar disks has focused on those in isolation from their environments. However, observations are now beginning to probe the earliest, most embedded phases of star formation, during which disks are rapidly accreting from their parent cores and cannot be modeled in isolation. We present a simple, one-zone model of protostellar accretion disks with high mass infall rates. Our model combines a self-consistent calculation of disk temperatures with an approximate treatment of angular momentum transport via two mechanisms. We use this model to survey the properties of protostellar disks across a wide range of stellar masses and evolutionary times, and make predictions for disks' masses, sizes, spiral structure, and fragmentation that will be directly testable by future large-scale surveys of deeply embedded disks. We define a dimensionless accretion-rotation parameter which, in conjunction with the disk's temperature, controls the disk evolution. We track the dominant mode of angular momentum transport, and demonstrate that for stars with final masses greater than roughly one solar mass, gravitational instabilities are the most important mechanism as most of the mass accumulates. We predict that binary formation through disk fission, fragmentation of the disk into small objects, and spiral arm strength all increase in importance to higher stellar masses.

Subject headings: accretion disks - binaries: general - stars: formation --ISM :clouds
\end{abstract}

\section{INTRODUCTION}

A young star system's visible T Tauri or Herbig stage is preceded by a deeply enshrouded phase of rapid accretion in which its character - multiplicity, disk properties, and tendency to form planets - is first forged. Although this embedded phase is likely the one during which most accretion onto the star occurs, the properties of disks during this period have received relatively little attention. This phase is difficult to model analytically because embedded disks are subject to large perturbations in the form of rapid accretion of mass and angular momentum, making local models and stability analyses problematic. Due to the high obscuration characteristic of this phase, disks are accessible primarily via radio and submillimeter observations, and such techniques provide limited sensitivity and angular resolution compared to what can be achieved for $\mathrm{T}$ Tauri and Herbig AE star disks using shorter wavelengths. Our knowledge of massive protostellar disks is particularly limited by this problem, since they do not have a significant unobscured phase, probably due to the destructive effects of ionizing radiation. These difficulties are compounded by the fact that massive stars form more rarely, and therefore tend to lie farther away. Detections of rotation and infall in a few systems hint at the presence of disks during the embedded phase, but are only preliminary (see recent reviews by Cesaroni et al. 2006, 2007, Beuther 2006, and Beuther et al. 2007).

While our knowledge of the embedded phase today is limited, it will soon come into sharp focus as new instru-

\footnotetext{
${ }^{1}$ Hubble Fellow
}

ments such as the Expanded Very Large Array (EVLA) and Atacama Large Millimeter Array (ALMA) become operational. In order to predict what these telescopes will discover about the formation of stars across a very broad mass range, $\sim 1-120 M_{\odot}$, we present evolutionary models of star-disk systems reacting to infall at very high rates. We concentrate our efforts on the physical processes that control disk evolution, such as the torque from a turbulent infall, the reprocessing of starlight by the infall envelope, and the character of the self-gravitational instabilities. The disk itself we model with a highly simplified, single-zone treatment. Although multidimensional simulations provide a much more detailed view of disk formation and evolution during the embedded phase (e.g. Goodwin et al. 2004 Krumholz et al. 2007b), their high computational cost and the limited range of physical processes they include mean that these simulations can explore only small regions of parameter space. They cannot easily make predictions across a broad range of stellar mass scales and evolutionary times. In contrast, our semi-analytic approach permits us to incorporate more physical effects and explore the consequences of environmental parameters more rapidly, and in a more systematic way. This serves two complementary ends: the theoretical goal of understanding angular momentum transport and fragmentation in the embedded phase, and the observational goal of making concrete predictions about the properties of young, massive disks.

Although we include a range of physical processes in our models; the most important in driving the evolution of embedded disks in our calculations is self-gravity. Selfgravity plays a central role in mediating angular momen- 
tum transport and triggering fragmentation into a binary or multiple system. Its importance in star formation has long been recognized (Larson 1984), and our study is preceded by evolutionary calculations which incorporate accretion and self-gravity into one-dimensional (Lin \& Pringle 1987, 1990; Nakamoto \& Nakagawa 1994, 1995, Hueso \& Guillot 2005) and two-dimensional (Vorobyov \& Basu 2005, 2006) simulations. Although lower in resolution, our approach is distinguished from these works in several ways:

$i$. In contrast to all one-dimensional calculations to date, we account for the dependence of gravitational torques on the disk-to-total mass ratio in addition to Toomre's instability parameter.

$i i$. We consider the possibility that disks will fragment if they become sufficiently unstable.

iii. We consider fluctuations of the vector angular momentum in the infall due to realistic turbulence in the collapsing cloud core.

$i v$. We employ a realistic model for the irradiation of the disk midplane, in which starlight is reprocessed at the inner wall of an outflow cavity while inflow is occurring.

$v$. We survey the conditions of intermediate-mass and massive star formation, rather than focusing exclusively on conditions in nearby low-mass star forming regions such as Taurus.

The first of these is important for all protostellar disks, since the disk mass is never negligible when the Toomre parameter is small. Features (ii) and (iii) are of primary importance in the formation of massive stars, which accrete from strongly turbulent regions (Myers \& Fuller 1992 McLaughlin \& Pudritz 1997) and which are likely to undergo disk fragmentation (Kratter \& Matzner 2006 hereafter KM06). Irradiation is most important for lowmass stars, whose disks it strongly stabilizes (Matzner \& Levin 2005), but it remains significant in massive star formation as well.

In $\$ 2$ we outline our model for the infall rate of matter and angular momentum. We develop a model for disk accretion and fragmentation in $\$ 3$. In $\$ 4$ we define the key environmental variables that control protostellar disk evolution and sketch a qualitative evolutionary sequence based on their fiducial values. In $\$ 5$ we present the results for our fiducial case, and explore the effect of varying our parameters. In $\$ 6$ we discuss the observable predictions that our model makes, and finally in $\S 7$ we summarize our main results.

\section{INFALL ONTO DISKS}

Since we are interested in the behavior of a disk that is subject to strong perturbations from its environment, we begin building our model by constructing a prescription for the infall of matter and angular momentum onto a disk. This accretion comes from a background "core" (Shu 1977; McLaughlin \& Pudritz 1997, McKee \& Tan 2003), whose properties and interaction with a disk we discuss in this section.

\subsection{Star Formation By Core Collapse}

We model the accretion of mass and angular momentum using the two-component core model of McKee \& $\operatorname{Tan}(2003)$, which is a generalization of the TNT (thermal plus non-thermal) model of Myers \& Fuller (1992). In this model, which we summarize here for convenience, the density distribution within a core follows a twocomponent power law distribution

$$
\rho=\rho_{s}\left(\frac{R_{\mathrm{c}}}{r}\right)^{-k_{\rho}}+\frac{c_{\mathrm{s}, \mathrm{c}}^{2}}{2 \pi G r^{2}},
$$

where $R_{\mathrm{c}}$ is the outer radius of the core, $c_{\mathrm{s}, \mathrm{c}}$ is the thermal sound speed within it (assumed to be constant), and $\rho_{s}$ is the density at the core's surface. We follow McKee \& Tan (2003) in adopting $k_{\rho}=1.5$ as the fiducial value of the turbulence-supported density index. Physically, the first term describes an envelope supported primarily by turbulent motions, while the second describes a thermally-supported region at its center. A model of this sort is fully specified in terms of the three parameters: the core mass

$$
M_{\mathrm{c}}=\frac{4 \pi}{3-k_{\rho}} \rho_{s} R_{\mathrm{c}}^{3}+2 \frac{c_{s, c}^{2} R_{\mathrm{c}}}{G},
$$

surface density

$$
\Sigma_{\mathrm{c}}=\frac{M_{\mathrm{c}}}{\pi R_{\mathrm{c}}^{2}},
$$

and temperature

$$
T_{\mathrm{c}}=\frac{m}{k_{B}} c_{s, c}^{2},
$$

where $m=3.9 \times 10^{-24} \mathrm{~g}$ is the mean particle mass in a gas of molecular hydrogen and helium mixed in the standard cosmic abundance. Observed regions of star formation contain cores with masses $\sim 1-100 M_{\odot}$, surface densities $\Sigma_{\mathrm{c}} \approx 0.03-1 \mathrm{~g} \mathrm{~cm}^{-2}$, and temperatures of 10 to $50 \mathrm{~K}$ (Johnstone et al. 2001; Plume et al. 1997).

The core is taken to be in approximate hydrostatic balance initially, and this condition specifies the required level of turbulent support. The non-thermal velocity dispersion in the shell at radius $r$ is

$$
\sigma(r)^{2}=\frac{2 \pi}{3 \phi_{B}\left(k_{\rho}-1\right)} \frac{G M(r)}{r}-c_{\mathrm{s}, \mathrm{c}}^{2}
$$

where $M(r)$ is the mass at radii of $r$ or less and $\phi_{B} \simeq 2.8$ approximately accounts for the magnetic contribution to the total pressure. Except when $M(r) \ll 1 M_{\odot}$ or $\Sigma_{c}<$ $0.1 \mathrm{~g} \mathrm{~cm}^{-2}$, the first term is much larger than the second, so that the velocity dispersion is primarily non-thermal.

Core collapse commences at time zero, and a mass shell initially at radius $r$ falls onto the disk in a time comparable to the free fall time evaluated at its initial density, $t_{\mathrm{ff}}(r)=[3 \pi / 32 G \rho(r)]^{1 / 2}$. In practice, we use the McKee \& Tan (2003) accretion rate approximation to determine $M$ on to the star-disk system as a function of the total core mass and the current amount of mass that has accreted: 
$\dot{M}_{\mathrm{in}} \approx\left(\frac{\phi_{*} M_{* f}}{t_{\mathrm{ff}, \mathrm{s}}}\right)\left[\left(\frac{M_{* d}}{M_{* f}}\right)^{2 q}+\left(\frac{\phi_{* \mathrm{th}}}{\phi_{* \mathrm{nth}}}\right)^{2}\left(\frac{\varepsilon M_{\mathrm{th}}}{M_{* f}}\right)^{2 q}\right]^{1 / 2}$

where $M_{* f}$ is the final disk plus stellar mass, $M_{* d}$ is the current disk plus stellar mass, $t_{\mathrm{ff}, \mathrm{s}}$ is the free-fall time evaluated at the core surface (i.e. at $\rho=\rho\left(R_{c}\right)$ ),

$$
\begin{aligned}
q & =\frac{3\left(2-k_{\rho}\right)}{2\left(3-k_{\rho}\right)}, \\
M_{\mathrm{th}} & =10^{-3.1}\left(\frac{T}{20 \mathrm{~K}}\right)^{3}\left(\frac{30 \varepsilon M_{\odot}}{M_{* f}}\right)^{1 / 2} \Sigma_{c, 0}^{-3 / 2} M_{\odot},
\end{aligned}
$$

$\Sigma_{\mathrm{c}, 0}=\Sigma_{\mathrm{c}} /\left(\mathrm{g} \mathrm{cm}^{-2}\right)$, and $\phi_{*}, \phi_{* \mathrm{nth}}$, and $\phi_{* \text { th }}$ are constants of order unity that depend on the polytropic index and magnetic field strength. The efficiency factor

$$
\varepsilon=\frac{M_{* f}}{M_{c}}
$$

represents the fraction of the core mass that lands on the star-disk system rather than being blown out by protostellar outflows. We again follow McKee \& Tan (2003) in adopting $\varepsilon=0.5$, a value typical of low-mass star formation (Matzner \& McKee 2000).

\subsection{Angular Momentum of the Infalling Material}

Equation (6) gives the mass infall rate $\dot{M}_{\text {in }}(t)$ from the core as a function of time. The second component of our core model is to specify the corresponding rate of angular momentum infall $\dot{\mathbf{J}}_{\text {in }}(t)$. We compute this in several steps. First, we approximate the vector specific angular momentum $\mathbf{j}(r)$ averaged over a shell of material at radius $r$ as described below. Then we compute $M(t) \equiv \int_{0}^{t} \dot{M}_{\text {in }}\left(t^{\prime}\right) / \epsilon d t^{\prime}$, the total mass from the core that has either fallen onto the star-disk system or been ejected at time $t$. From the core density profile (equation 11 we also compute $M(r) \equiv \int_{0}^{r} 4 \pi r^{\prime 2} \rho\left(r^{\prime}\right) d r^{\prime}$, the mass of the initial core enclosed within radius $r$. Assuming the core accretes inside-out, we set $M(r)=M(t)$ and solve for $r(t)$, which gives the initial radius $r$ of the shell of mass that arrives at the star-disk system at time $t$. Assuming that the specific angular momentum of the gas does not change before it reaches the disk, the angular momentum accretion rate is then simply given by $\dot{\mathbf{J}}_{\text {in }}(t)=\dot{M}_{\text {in }}(t) \mathbf{j}(r(t))$.

The remaining step is to specify how we estimate $\mathbf{j}(r)$. Star forming cores are often modeled as solid body rotators characterized by the ratio $\beta$ of rotational to gravitational energy, but we adopt a more realistic model in which turbulent fluctuations affect the infalling gas. Following Burkert \& Bodenheimer (2000), Fisher (2004), Matzner \& Levin (2005) and KM06, we assume that the observed angular momenta of cores (Goodman et al. 1993 ) can be modeled using an idealized turbulent velocity field. Using the method of Dubinski et al. (1995), we generate a numerical realization of an isotropic, homogeneous, Gaussian random velocity field $\mathbf{v}(\mathbf{r})$. We require that the power spectrum $P(k)$ of this turbulent field reproduce the scalings required by turbulent support against gravity: $\sigma(r)^{2} \propto G M(r) / r \propto r^{2-k_{\rho}}$ at large radii, so that $\sigma(r) \propto r^{1 / 4}$ for $k_{\rho}=3 / 2$. Parceval's theorem or dimensional analysis then require $P(k) \propto k^{-3 / 2}$.

We note that numerical simulations of supersonic turbulence consistently show the steeper spectral index -2 (Porter et al. 1992) which is understood as the spectrum of an individual shock and as the exact limit of Burgers turbulence. Matzner (2007) and Nakamura \& Li (2007) have shown that a shallower index is expected when turbulence is driven by protostellar outflows, however, and our chosen power spectrum is consistent with the line width-size relation for massive cores (e.g., Caselli \& Myers 1995; Plume et al. 1997). Our homogeneous velocity field is surely an idealization, but not a grave one.

After scaling our numerical domain to match the core radius $R_{c}$, we normalize $\mathbf{v}$ such that the one-dimensional velocity dispersion of a spherical shell with this radius equals $\sigma(r)$ defined in equation (5). In practice we fit $R_{c}$ within a $256^{3}$ section of a $1024^{3}$ grid of velocities, because periodicity causes artifacts on scales larger than about $1 / 4$ of the box size. From this field we calculate the specific angular momentum $\mathbf{j}=\mathbf{r} \times \mathbf{v}$ at every point and the mean specific angular momentum $\mathbf{j}(r)$ in a shell at radius $r$. Note that KM06 calculate the expected magnitude and dispersion of $\mathbf{j}(r)$ for velocity fields of precisely this type; our results agree with their predictions to about $50 \%$, which is within the scatter they predict.

\section{DYNAMICS OF THE DISK}

\subsection{Approach to disk evolution}

Given the rate at which mass and angular momentum accrete, we must calculate the reaction of the disk. At any given time, our star-plus-disk system is characterized by the disk mass $M_{d}$, the central star mass $M_{*}$, and the total angular momentum content of the disk $\mathbf{J}_{d}$. Given these quantities, and the rates of mass and angular momentum infall $\dot{M}_{\text {in }}$ and $\dot{\mathbf{J}}_{\text {in }}$, we wish to compute the time rate of changes $\dot{M}_{d}, \dot{M}_{*}$, and $\dot{\mathbf{J}}_{d}$.

Using the separation between the thermal, orbital, and accretion timescales, we assume our disks are in a thermal steady state and draining at a rate determined by their current global parameters. We shall later refer to this as the assumption of thermal and mechanical equilibrium.

In $\S 3.2$, we estimate the disk accretion rate onto the central star due to various angular momentum transport mechanisms. In $\S 3.3$ we discuss thermal equilibrium in the disk, which together with the aforementioned condition of mechanical equilibrium allows us to selfconsistently compute the accretion rate from the disk to the star $\dot{M}_{*}$. In $\S 3.4$ we describe the corresponding angular momentum evolution $\dot{\mathbf{J}}_{d}$. Finally, in $\S 3.5$ and $\S 3.6$, we discuss our prescriptions for disk fragmentation and binary formation.

It is helpful before proceeding to define two dimensionless parameters that characterize the strength of the disk's self-gravity. These are the disk-to-total mass ratio

$$
\mu=\frac{M_{d}}{M_{d}+M_{*}}
$$

and Toomress (1964) instability parameter

$$
Q=\frac{c_{s} \kappa}{\pi G \Sigma_{d}}
$$


where $\kappa$ is the radial epicyclic frequency, $c_{s}$ speed of density waves, and $\Sigma_{d}$ is the disk's mass surface density. In practice we evaluate $Q$ using $\kappa \rightarrow \Omega=\left(G M_{\text {tot }} / R_{d}^{3}\right)^{1 / 2}$, the total orbital frequency, since the difference between them is only marginally significant even when the disk mass is quite large. Here $R_{d}=j_{d}^{2} / G M_{\text {tot }}$. We also approximate $c_{s}$ using the isothermal sound speed. To characterize gravitational instability, we use the minimum value of $Q$ - the value at $R_{d}$, the outer boundary of our active disk. In this evaluation we assume a profile $\Sigma_{d} \propto r^{-1}$ (a choice we justify in $\S 3.4$ ) so that $\Sigma_{d}\left(R_{d}\right)=M_{d} /\left(2 \pi R_{d}^{2}\right)$, and we evaluate $c_{s}$ and $\Omega$ at the edge of the disk.

We base our models on the fundamental assumption that the self-gravitational behavior of an accretion disk depends primarily on the structural parameters $\mu$ and $Q$ - so that its evolution is controlled by heating and cooling $(\S 3.3)$, which alter $Q$, and accretion onto and through the disk, which alters $\mu$. This approach permits us to treat the disk's mechanical and thermal properties separately, before combining them into a model for its evolution. This division also guides our use of published work, since it implies that simulations with adiabatic equations of state and those with an imposed cooling rate may be combined into a mechanical model for disk evolution, which we may then use to model irradiated protostellar disks. Finally, it prompts us to treat the onset of disk fragmentation and disk fission as boundaries in the space of $\mu$ and $Q$, rather than in terms of a critical cooling rate (which is the natural and conventional description for simulations that include cooling but not irradiation). In $\S 3.5$ we argue that these descriptions are effectively equivalent.

Models based on this assumption are guaranteed to be somewhat approximate, because a disk's mechanical evolution must, at some level, reflect additional parameters: its dimensionality, its equation of state, the specifics of its heating and cooling processes, and the magnitude of external perturbations (like tides), to name a few. However we expect our results to be valid, both because we believe that $\mu$ and $Q$ are indeed the most significant parameters for gravitational instability, and because our model is calibrated to realistic numerical models. Additional simulations will be required to test this approach.

\subsection{Angular Momentum Transport and Disk Accretion}

A key element of our model is a prescription for angular momentum transport and the rate $\dot{M}_{*}$ at which matter accretes onto the central star - or more specifically, the dimensionless rate $\dot{M}_{*} /\left(M_{d} \Omega\right)$. In practice we first construct a model for an effective Shakura \& Sunyaev $\alpha$ parameter, which we define through the steady-state relation

$$
\dot{M}_{*}=\frac{3 \alpha c_{s}^{3}}{G Q}
$$

so that

$$
\frac{\dot{M}_{*}}{M_{d} \Omega}=\frac{3 \alpha Q^{2}}{8} \mu^{2},
$$

where the factor of of $3 / 8$ comes from the assumption that the disk surface density falls as $r^{-1}$. We do not mean to imply by this that transport induced by gravitational instability is purely local (Balbus \& Papaloizou 1999), although this does appear to be the case for sufficiently thin and light disks (Gammie 2001: Lodato \& Rice 2005).

We divide $\alpha$ into two contributions: $\alpha_{\mathrm{MRI}}$, due to the magnetorotational instability (MRI), and $\alpha_{\mathrm{GI}}$, due to gravitational instability. In keeping with the strategy described in $\S 3.1$, we consider $\alpha_{\text {GI }}$ to be a pure function of $\mu$ and $Q$. We combine it and the MRI contribution linearly:

$$
\alpha=\alpha_{\mathrm{GI}}+\alpha_{M R I}
$$

We create our model for $\alpha_{G I}(Q, \mu)$ using results from the simulations of Laughlin \& Rozyczka (1996), Rice et al. (2003), Lodato \& Rice (2004), Lodato \& Rice (2005) and Gammie (2001). We adopt a constant value for $\alpha_{M R I}$, as discussed below.

\subsubsection{Overview of Simulations}

The three sets of simulations span a large fraction of our parameter space in $Q$ and $\mu$. The global disk simulations of Laughlin \& Rozyczka (1996) explore $Q>1$ and non-negligible values of $\mu$ using a two-dimensional hydrodynamic, self-gravity code; they supress local fragmentation by imposing an adiabatic equation of state. The simulations of Gammie (2001) represent the limit $\mu \rightarrow 0$, for values of $Q$ which approach unity from above, and are most directly applicable to quasar disks. Gammie imposes cooling with a fixed cooling time, $\tau_{c}$, which is proportional to the orbital time. He finds a regime of steady gravity-induced turbulence, for disks that cool over many orbits. If $\tau_{c}$ is too short $\left(<3 \Omega^{-1}\right)$, however, the disk fragments as $Q$ drops below unity. The disk viscosity is highest at the boundary of fragmentation. Angular momentum transport in this regime is quite local, with an effective value of $\alpha$ that is inversely proportional to the cooling rate. Our third source is the global SPH simulations of Rice et al. (2003), Lodato \& Rice (2004), and Lodato \& Rice (2005), in which a cooling time $\propto \Omega^{-1}$ is imposed locally; these cover the entire parameter space in $\mu$. In these simulations $Q$ is initially 2 , but it descends towards unity. Here again, the disk fragments if $\Omega \tau_{c}$ is too small, although the critical value of this parameter is different than Gammie found.

\subsubsection{Accretion Model}

To derive a relatively simple analytic fit to the simulation data, we must extract a characteristic $\alpha_{\mathrm{GI}}, Q$, and $\mu$ from the simulations listed above. Because the numerical approaches are varied, we are unable to use the same method for each. The values are derived as follows for each type of simulation:

1. We estimate $\alpha_{\mathrm{GI}}$ from Laughlin \& Rozyczka (1996) using their equations 24-26; $Q$ and $\mu$ are given.

2. Values of $\alpha$ from Rice et al. (2003), Lodato \& Rice (2004), Lodato \& Rice (2005) are taken directly from plots, when available. Because $\alpha$ varies with radius, we take an approximate value from the outer region of their disk before the density begins to fall off steeply. When plots are not available, we use the critical value of $\tau_{c}$ to calculate $\alpha_{\mathrm{GI}}$ at the fragmentation boundary, which we take to be $Q=1$ (see $\S 3.5$ ). Again, $\mu$ is given. 
3. Gammie (2001) provides one value of $\alpha_{\mathrm{GI}}$ at $Q=1$ for a disk with $\mu \rightarrow 0$, which we adopt.

These values of $\alpha_{\mathrm{GI}}$ are shown in Figure 1 according to the estimated values of $\mu$ and $Q$ that accompany each of them. We treat them as a data set to be fit within our analytical model for $\alpha_{\mathrm{GI}}$, which is displayed in the underlying contours in that figure. Imposing the realistic condition that $\alpha_{\mathrm{GI}}$ is continuous and equals zero for $Q>$ 2 (when the gravitational instability should shut off as suggested by Griv (2006)), we find that two components are required:

$$
\alpha_{G I}=\left(\alpha_{\text {short }}^{2}+\alpha_{\text {long }}^{2}\right)^{1 / 2}
$$

where

$$
\alpha_{\text {short }}=\max \left[0.14\left(\frac{1.3^{2}}{Q^{2}}-1\right)(1-\mu)^{1.15}, 0\right]
$$

and

$$
\alpha_{\text {long }}=\max \left[\frac{1.4 \times 10^{-3}(2-Q)}{\mu^{5 / 4} Q^{1 / 2}}, 0\right] .
$$

In fact we apply equation (15) only to the region $Q>1$. Because we expect the gravitational torque to saturate when fragmentation occurs, we hold $\alpha$ constant, for a given $\mu$, when $Q<1$; this amounts to replacing $Q \rightarrow \max (Q, 1)$ in the above equations. This has no practical consequences for our calculations, however, since our treatment of fragmentation $(\S 3.5)$ prevents our disks from sampling values of $Q$ much below unity.

Our nomenclature in equation 15 reflects our interpretation. The "short" component $\alpha_{\text {short }}$ dominates for $Q \lesssim 1.3$, hence for relatively thin disks. We think of it as arising from modes with relatively high spatial wavenumbers and short wavelengths (Lodato \& Rice 2004, 2005). Note that its functional form resembles the model of Lin \& Pringle (1990) (their eq. 16) modified by a mild $\mu$ dependence, which is comparable to the scale height dependence derived in equation (2.5) of Lin \& Pringle (1987).

The "long" component $\alpha_{\text {long }}$ is important in thicker disks whose instabilities are likely to be dominated by loosely wound, $m=2$ spiral patterns. We require it because we include the adiabatic simulations of Laughlin \& Rozyczka (1996), which sample higher values of $Q$ because they cannot cool. (Indeed, $Q$ rises during these simulations.) Our fundamental assumption ( $\$ 3.1$ leads us to incorporate these results into a single model for $\alpha(\mu, Q)$, despite the difference in thermal physics. Future simulations can test this assumption by imposing heating (via irradiation, say) as well as cooling: our model implies that the derived $\alpha_{\text {GI }}$ will be comparable to Laughlin $\&$ Rozyczkals, when $Q$ and $\mu$ take similar values. While simulations such as Boley et al. (2006) and Cai et al. (2007) are making dramatic process towards accurately modelling heating, cooling, and irradiation, a wider parameter space is necessary for comparison. We note that Sellwood \& Carlberg (1984) and Griv (2006) also find non-axisymmetric instabilities for massive disks with $Q$ in the range $1.3-2$.

As shown in Figure 1 our model for $\alpha_{\mathrm{GI}}$ agrees reasonably well with data from the simulations, though we fail to fit a couple points at very high $\mu$ and low $Q$. Note that $\alpha_{\mathrm{GI}}$ for these points from Laughlin \& Rozyczka (1996) are uncertain themselves.

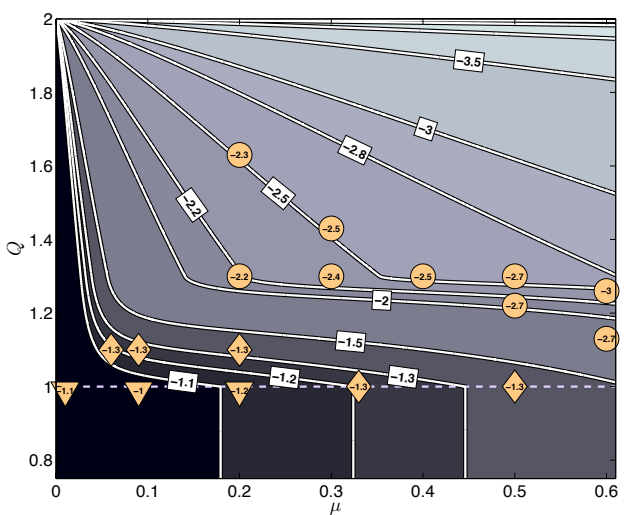

FIG. 1.- Contours of the viscosity parameter $\log \left(\alpha_{\mathrm{GI}}\right)$ due to gravitational instabilities (eq. 15; white squares are contour labels. Results from numerical simulations are marked with circles, diamonds, and triangles. Circles show simulations with adiabatic equations of state (Laughlin \& Rozyczka 1996), diamonds show simulations with an imposed cooling rate that reach steady state (Lodato \& Rice 2004 2005), and triangles show the maximum $\alpha_{\mathrm{GI}}$ achieved in simulations with imposed cooling that probe the fragmentation boundary (Gammie 2001 Rice et al. 2003 Lodato \& Rice 2004 2005). Note that the point at $\mu=0$ corresponds to the purely local simulation of Gammie (2001). The $Q=1$ boundary is marked with a dashed line.

It is important to bear in mind that our accretion model is only a rough representation of the numerical results on which it is based, and that it can be improved as more simulations become available. For instance, we place no stock in the weak divergence of $\alpha_{\text {long }}$ as $\mu \rightarrow 0$ : this feature is a product of our fit to numerical results at larger $\mu$, and it would be an unwise extrapolation to use our model for disks with very low $\mu$ and moderate $Q$. It does not affect our results, as our disks do not sample this regime.

Finally, we assume the disk is sufficiently ionized to support magnetic turbulence, and we represent the MRI with the constant value $\alpha_{\mathrm{MRI}}=10^{-2}$. The typical value of $\alpha_{\mathrm{MRI}}$ is rather uncertain; see Pessah et al. (2007) for a synthesis of recent work, and Hueso \& Guillot (2005) for a recent consideration of observational constraints in low-mass protostellar disks. Gravitational torques exceed those from the MRI for much of the accretion phase. We discuss the influence of $\alpha_{\mathrm{MRI}}$ on our results in $\S 5.5$

Figure 2 illustrates our model for the dimensionless accretion rate $\dot{M}_{*} /\left(M_{d} \Omega\right)$ as a function of $Q$ and $\mu$. We draw attention to several key features of the plot. First, note that at low $Q$ there is a tongue-like feature that increases in intensity with increasing disk mass. This is due to the strong dependence of $\alpha_{\text {short }}$ on both $Q$ and $\mu$ At higher values of $Q$ and lower values of $\mu$ the contours steepen due to the weak divergence of $\alpha_{\text {long }}$ as $\mu \rightarrow 0$, which is probably not physical. The curvature towards higher $Q$ and $\mu$ shows the dominance of the MRI for $Q>2$. The fact that the dimensionless accretion rate takes numerical values up to $10^{-2.4}$, with a typical values $\sim 10^{-3.5}$, implies that massive disks drain on timescales ranging from $\sim 40$ to a few thousand orbits, with five hundred orbits being typical.

\subsection{Disk Thermal Equilibrium}

We have now specified the rate at which the disk accretes onto a central star as a function of $Q$ and $\mu$. How- 


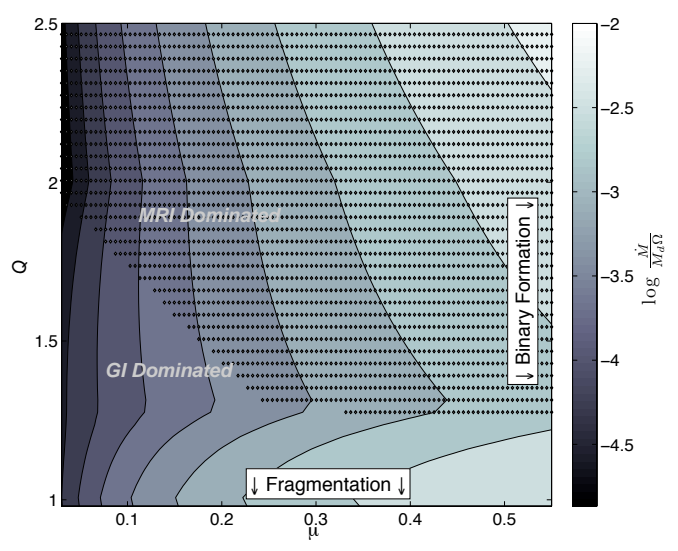

Fig. 2.- Contours of the dimensionless accretion rate from the disk onto the star $\left(\dot{M}_{*} / M_{d} \Omega\right)$ from all transport components of our model. The lowest contour level is $10^{-4.8}$, and subsequent contours increase by 0.3 dex. The effect of each transport mechanism is apparent in the curvature of the contours. At $Q<1.3$ the horizontal "tongue" outlines the region in which short wavelength instability dominates accretion. The more vertical slope of the contours at lower $\mu$ and $Q>1.3$ shows the dominance of the long wavelength instability. The MRI causes a mild kink in the contours across the $Q=2$ boundary and is more dominant at higher disk masses due to our assumption of a constant $\alpha$ : equation 13 illustrates that a constant $\alpha$ will cause higher accretion rates at higher values of $\mu$.

ever, this does not fully specify the accretion rate, because while $\mu$ may be directly computed from our "primitive" variables $M_{d}, M_{*}$, and $\mathbf{J}_{d}$, the Toomre stability parameter $Q$ cannot be, because it depends on the sound speed $c_{s}$ and thus the temperature within the disk. We can determine this by requiring that the disk be in thermal equilibrium.

To compute the disk's thermal state, we follow the approach of KM06, in which disks are heated by a combination of stellar irradiation and viscous dissipation due to accretion. In equilibrium, the disk midplane temperature satisfies the approximate relation

$$
\sigma T^{4}=\left(\frac{8}{3} \tau_{R}+\frac{1}{4 \tau_{P}}\right) F_{v}+F_{\mathrm{irr}},
$$

where $F_{v}$ is the rate of viscious dissipation per unit area in the disk, $F_{\text {irr }}$ is the flux of starlight (whether direct or reprocessed) onto the disk surface, and $\tau_{R, P}=\kappa_{R, P} \Sigma / 2$ are the Rosseland and Planck optical depths to the midplane. The viscous dissipation per unit area is

$$
F_{v}=\frac{3 \dot{M} \Omega}{8 \pi}
$$

and we compute the opacities using the Semenov et al. (2003) model for $\kappa_{R, P}(T)$ : in particular, we use their homogeneous-aggregate dust model with normal silicates, calculated at the appropriate density.

Low-mass stars' luminosities are accretion-dominated in the main accretion phase, but those above about 15 $M_{\odot}$ reach the zero-age main sequence (ZAMS) while still accreting. To include both accretion luminosity and other sources in our calculation of $F_{\text {irr }}$, we use the protostellar evolution code of Krumholz \& Thompson (2007), based on the McKee \& Tan (2003) protostellar evolution model, to compute the luminosity $L_{*}$ of the central star as a function of its accretion history. The model includes contributions to the protostellar luminosity from accretion on the stellar surface, Kelvin-Helmholtz contraction, and, once the temperature rises high enough, deuterium and then hydrogen burning.

During the infall, dust within the infall envelope reprocesses starlight and casts it down on the disk. By performing ray tracing within an inflow envelope with a central outflow cavity, Matzner \& Levin (2005) determine the fraction of light received by the disk assuming the infall envelope is optically thick to the stellar radiation, and optically thin to its own IR re-radiation: they find

$$
F_{\text {irr }}=\frac{0.1}{\varepsilon^{0.35}} \frac{L_{*}}{4 \pi R_{d}^{2}} .
$$

The weak dependence on the accretion efficiency $\varepsilon$ arises from a picture in which the outflow clears a fraction $1-\varepsilon$ of the core, so that infall streamlines originate from regions separated from the axis by angles $\theta$ such that $\cos \theta>\varepsilon$. Recently, Rodríguez et al. (2005) have observed an outflow near an O-type protostar with an opening angle of approximately $25^{\circ}$; this is in reasonable agreement with the model chosen here, since infall occurs at wider angles.

Once the core has accreted entirely and the envelope can no longer re-process starlight, we make an (unrealistically) abrupt switch to a model in which $\dot{M}_{\text {in }}=0$. The star continues to acquire mass from the disk, which represents a non-negligible reservoir. From this point on we calculate $F_{\text {irr }}$ in the manner of Chiang \& Goldreich (1997). We first identify the fraction of $L_{*}$ intercepted by the surface which is optically thick to stellar photons, assuming for this purpose that $H \propto R^{9 / 7}$ and that the dust density is a Gaussian, of scale height $H$, in the height above the midplane. We also calculate the equilibrium temperature of dust in this reprocessing layer. We then calculate $F_{\text {irr }}$ as that fraction of the reprocessed radiation which is reabsorbed by the disk, allowing for the possibility that the disk will be optically thin at the relevant wavelengths. We find the reprocessing height is slightly larger than a scale height $(1.5 \mathrm{H}$ being typical); higher values are typical of more massive disks, which are more opaque.

Though negligible during the accretion phase, we also include a background radiation field due to the cloud (modeled as an optically thin dust layer) and the cosmic microwave background. This prevents disks from becoming unrealistically cold at large radii and late times. Our cloud irradiation serves as a stand-in for one neglected heat source in clusters: irradiation from surrounding stars. This effect is likely important for (a) very dense regions, and (b) late times when disk radii stretch out to $10^{4} \mathrm{AU}$. Due to the wide variance in the strength of this effect, we do not address heating by neighbors here. There is also minor heating due to the accretion shock that feeds the disk; however, KM06 have argued that this is negligible in general.

While our background heating is only important at late times, we do not report results for $t>2 \mathrm{Myr}$ as this may exceed the lifetime of gas disks, even the low-mass ones (Jayawardhana et al. 2006). The uncertainties in our procedure therefore have little effect on the results we obtain.

We have now fully specified the conditions of thermal 
and mechanical equilibrium for this disk, and we can use them to compute the accretion rate. Equations $\sqrt{13}$ and (18) constitute two equations for the unknowns $Q$ and $\dot{M}_{*}$. For any given $M_{d}, M_{*}$, and $\mathbf{J}_{\mathbf{d}}$, we may solve them to determine $\dot{M}_{*}$. This in turn also specifies the rate of change of the disk mass

$$
\dot{M}_{d}=\dot{M}_{\text {in }}-\dot{M}_{*} .
$$

Note that $M_{d}$ can also be modified by disk fragmentation and binary formation, as described in $\S 3.5$ and $\S 3.6$.

\subsection{An Outer Disk and the Braking Torque}

When describing standard steady-state disks, one implicitly assumes that when angular momentum is transported radially, it travels out to large radii in an insignificant amount of mass. In our current model, we effectively keep track of an "inner" disk: the portion containing the majority of the mass. This justifies our choice of surface density profile $\Sigma \propto r^{-1}$, since the radius at which this power-law slope is achieved is also the radius that encloses most of the mass. We allow for a small amount (2\%) of material raining in from the core to be carried out with the angular momentum.

The disk's angular momentum is then equal to that of the infalling material, in addition to the amount already in the disk, minus some portion which has been transferred to this outer disk. The disk loses a fraction $b_{j}$ of its angular momentum and a small amount of mass on the viscous timescale $\tau_{v}=M_{d} / \dot{M}_{*}$, so long as matter is still accreting from the core:

$$
\dot{\mathbf{J}}_{d}=\mathbf{j}_{\text {in }} \dot{M}_{\text {in }}-b_{j}\left(\frac{\dot{M}_{\text {in }}}{\dot{M}_{*}}\right) \frac{\dot{M}_{\text {in }}}{M_{d}} \mathbf{J}_{d}
$$

As above, the subscript "in" denotes newly accreted matter. The factor $\left(\dot{M}_{\mathrm{in}} / \dot{M}_{*}\right)$ is roughly unity in the main accretion phase, but goes to zero when accretion stops. We thus assume the outer disk only applies a torque when it gains matter from the inner disk. Without accretion the outer disk has no effect, and thus after accretion ends, the inner disk is free to expand self-similarly at constant $\mathbf{J}_{d}$. We consider this a conservative approach, considering that we do not treat effects like photoionization that might remove material from the inner and outer disk, especially in massive stellar clusters.

We consider $b_{j}=0.5$ to be typical; in this case an accreting disk loses about half its angular momentum each viscous time. Since the disk sheds mass at the same rate, this allows its radius to remain comparable to the circularization radius of the infalling material. Although our choice of $b_{j}$ is somewhat arbitrary we demonstrate that our parametrization makes the disk evolution somewhat independent of this value. See $\$ 5.6$ for discussion.

\subsection{Disk Fragmentation}

Since we have now computed $\dot{M}_{d}, \dot{M}_{*}$, and $\dot{\mathbf{J}}_{d}$, our model is almost complete. However, as demonstrated by both previous analytic work (KM06) and numerical simulations (Krumholz et al. 2007b; Vorobyov \& Basu 2006 , Lodato \& Rice 2005), our parameter space extends deeply into the regime where disk fragmentation is expected. We must account for this to model disk evolution. It is not our intent to follow the detailed evolution of the fragments formed, nor their mass spectrum; we are interested primarily in how they help the disk regulate $Q$.

In keeping with the approach outlined in $\S 3.1$, we make the important assumption that the disk fragments into small objects when $Q$ drops below a critical value, $Q_{\text {crit }}$, which we take to be unity. Other authors Gammie 2001; Rice et al. 2003) have pointed out the importance of a disk's thermal physics in setting the fragmentation boundary. In particular they find, in simulations with imposed cooling, a critical value of $\tau_{c} \Omega$ above which disks do not fragment, and below which they do.

Our fragmentation model reproduces these results (indeed, it is calibrated to the same simulations) and we believe that the two views are in fact equivalent. Within our model, a disk whose $Q$ is close to unity will be heated by accretion at a rate close to the critical cooling rate found in these simulations. In the absence of any additional heating, the cooling rate must exceed the critical value in order for $Q$ to fall below unity, so that fragmentation can commence. In other words, since in our model $Q$ is calculated based on the competition between cooling and the combination of viscous dissipation and irradiation, if $Q$ falls below unity then it is necessarily the case that the cooling rate is sufficient to overwhelm viscous heating, and therefore to satisfy a cooling condition similar to those identified by Gammie (2001) and Rice et al. (2003).

The benefit of our fragmentation model is that it can be easily extended into the realistic regime of irradiated disks, whereas a model that refers solely to the cooling time cannot.

We note, in support of our model, that we know no examples of disks for which $Q<1$ that do not fragment, nor those with $Q>1$ that do. Moreover, Rice et al. (2003) note that a sufficiently slowly cooling disk reaches an equilibrium at a $Q$ value higher than unity; this is consistent with a heating rate that drops sharply as $Q$ increases, as our accretion model would predict.

To implement fragmentation within our numerical models, we must specify how much mass goes into fragments each time step when $Q<1$. We first define a critical density, $\Sigma_{d, c}$ :

$$
\Sigma_{d, c}=\frac{c_{s} \Omega}{\pi G Q_{\mathrm{crit}}} ;
$$

a reduction of surface density from $\Sigma_{d}$ to $\Sigma_{c}$ would return the disk to stability. Because we expect fragmentation to happen over a dynamical time, we assume that it depletes the disk surface density at the rate:

$$
\dot{\Sigma}_{\text {frag }}=-\left(\Sigma_{d}-\Sigma_{d, c}\right) \Omega
$$

This rate is fast enough to ensure that $Q$ never dips appreciably below $Q_{\text {crit }}$.

For simplicity, we assume that while fragments contribute to the mass of the disk, they do not enter in Toomre's stability parameter $Q$ except insofar as they contribute to the binding mass. (One could consider a composite $Q$ : Rafikov 2001.) Nor do we follow the migration of fragments in the disk. Instead, we allow them to accrete onto the central star at the rate

$$
\dot{M}_{*, \text { frags }}=\phi_{f} M_{\text {frag }} \Omega,
$$


with $\phi_{f}=0.05$. The assumption is simply that some fraction of the fragments accrete each orbit. Fragments form preferentially at large distances from the star, and thus only a small amount of the fragment mass will make it into the central star each orbit. Changing this parameter by an order of magnitude only marginally alters the disk evolution.

We also make the important assumption that disks will always fragment to maintain stability, and allow accretion to proceed. While this is likely a good assumption based on the existence of massive stars that appear to have formed via disk accretion, the persistence of rapid accretion during fragmentation has not been satisfactorily demonstrated in numerical simulations. See 77.1

\subsection{Binary Formation}

A majority of stars, especially massive stars, are found in binary and multiple systems. Though we present a very simplified scenario for star formation, we do account for the possibility that a single secondary star will form if $M_{d}>M_{*}$, that is, if the disk grows unphysically large with respect to the central star. (As we discussed in $\S 3.4$. this may well be conservative - in the sense that secondaries may form at even lower values of $\mu$, or at earlier times through core fragmentation as described in Bonnell et al. 2004.) When $\mu>0.5$, we remove the excess mass and store it (and the associated angular momentum) in a binary star. Because this tends to happen before the disks have become very extended, we assume the binary separation will be small; we therefore ignore the binary as a source of angular momentum for the disk. As with fragments, we assume the disk is affected by the secondary star only through the increased binding mass. We make no attempt to account for its contribution to the total luminosity.

\subsection{Summary of Model}

We summarize our model via the flowchart shown in Figure 3, which illustrates a simplified version of the code's decision tree. At a given time $t$ we know the current disk and star mass, and the current angular momentum and mass infall rates as prescribed in \$2.1 and \$2.2. We can calculate $R_{d}$ and $\Sigma_{d}$ directly, and find the appropriate stellar luminosity based on its evolution, current mass, and accretion rate. Using these variables we self-consistently solve for the appropriate temperature, $Q$, and disk accretion rate as described in 83.3 With this information in hand, we determine whether the disk is stable, locally fragmenting, or forming a binary. If the disk is stable, we proceed to the next iteration. If $Q<1$, then the disk puts mass into fragments according to equation 23]. If $\mu>0.5$ we consider binary formation to have occurred, and the net angular momentum and disk mass over the critical threshold is placed into a binary (see \$3.6). We stop simulations after $2 \mathrm{Myr}$ for two reasons: first, the most massive stars in our parameter space are significantly evolved and so our stellar evolution models are no longer sufficient; and second, because many other effects begin to dominate the disks appearance at late stages due to gas-dust interaction and photo-evaporation (Keto 2007).

\section{EXPECTED TRENDS}

Before examining the numerical evolution, it is useful to make a couple analytical predictions for comparison.

First, can we constrain where disks ought to wander in the plane of $Q$ and $\mu$ ? This turns out to depend critically on the dimensionless system accretion rate

$$
\Re_{\text {in }} \equiv \frac{\dot{M}_{\text {in }}}{M_{* d} \Omega\left(R_{\text {circ }}\right)}=\frac{\dot{M}_{\text {in }} j_{\text {in }}^{3}}{G^{2} M_{* d}^{3}}
$$

which is the ratio of the mass accreted per radian of disk rotation (at the circularization radius $R_{\text {circ }}$ ) to the total system mass $M_{* d}=M_{*}+M_{d}$. Since the active inner disk has a radius comparable to $R_{\text {circ }}$, this controls how rapidly the disk gains mass via infall.

The importance of $\Re_{\text {in }}$ is apparent in the equation governing the evolution of the disk mass ratio $\mu$ :

$$
\begin{aligned}
\frac{\dot{\mu}}{\mu \Omega} & =\frac{\dot{M}_{\text {in }}}{M_{* d} \Omega}\left(\frac{1}{\mu}-1\right)-\frac{\dot{M}_{*}}{M_{d} \Omega} \\
& =\frac{\Omega\left(R_{\text {circ }}\right)}{\Omega}\left(\frac{1}{\mu}-1\right) \Re_{\text {in }}-\frac{\dot{M}_{*}}{M_{d} \Omega} .
\end{aligned}
$$

Since we consider $\dot{M}_{*} /\left(M_{d} \Omega\right)$ to be a function of $\mu$ and $Q$, we must know the disk temperature to solve for $\mu(t)$. Regardless, equation (27) shows that larger values of $\Re_{\text {in }}$ tend to cause the disk mass to increase as a fraction of the total mass. We may therefore view $\Re_{\text {in }}$ and $Q$ as the two parameters that define disk evolution - of which $\Re_{\text {in }}$ is imposed externally and $Q$ is determined locally.

Moreover, $\Re_{\text {in }}$ takes characteristic values in broad classes of accretion flows, such as the turbulent core models we employ. Suppose the rotational speed in the precollapse region is a fraction $f_{K}$ of the Kepler speed, so that $j_{\text {in }}=f_{K} r v_{K}(r)=f_{K}\left[G r M_{c}(r)\right]^{1 / 2}$, and suppose also that the mass accretion rate is a fraction $\varepsilon f_{\text {acc }}$ of the characteristic rate $v_{K}(r)^{3} / G$. Then,

$$
\Re_{\mathrm{in}}=\frac{f_{K}^{3} f_{\mathrm{acc}}}{\varepsilon^{2}}
$$

(In this expression, negative three powers of $\varepsilon$ appear because the binding mass is $\varepsilon$ times smaller for the disk than for the core; one of these is compensated by the reduction of the accretion rate.)

In $\S 2.1$ we adopted the McKee \& Tan (2003) model for massive star formation due to core collapse of a singular, turbulent, polytropic sphere in initial equilibrium. Their equations (28), (35), and (36) imply

$$
f_{\text {acc }}=0.84\left(1-0.30 k_{\rho}\right)\left(\frac{3-k_{\rho}}{1+H_{0}}\right)^{1 / 2}
$$

within $2 \%$, where $1+H_{0} \simeq 2$ represents the support due to static magnetic fields ( $\mathrm{Li} \& \mathrm{Shu} 1996)$. (Note, their equation [28] is a fit made by Mckee \& Tan 2002 to the results of McLaughlin \& Pudritz 1997.)

KM06 predicted the turbulent angular momentum of these cores; our parameter $f_{K}$ equals $\left(\theta_{j} \phi_{j}\right)^{1 / 2}$ in their paper. Their equations (25), (26), and (29) imply

$$
f_{K}=\frac{0.49}{\phi_{B}^{1 / 2}} \frac{\left(1-k_{\rho} / 2\right)^{0.42}}{\left(k_{\rho}-1\right)^{1 / 2}},
$$

with excursions upward by about $50 \%$ and downward by about a factor of three expected around this value; here 


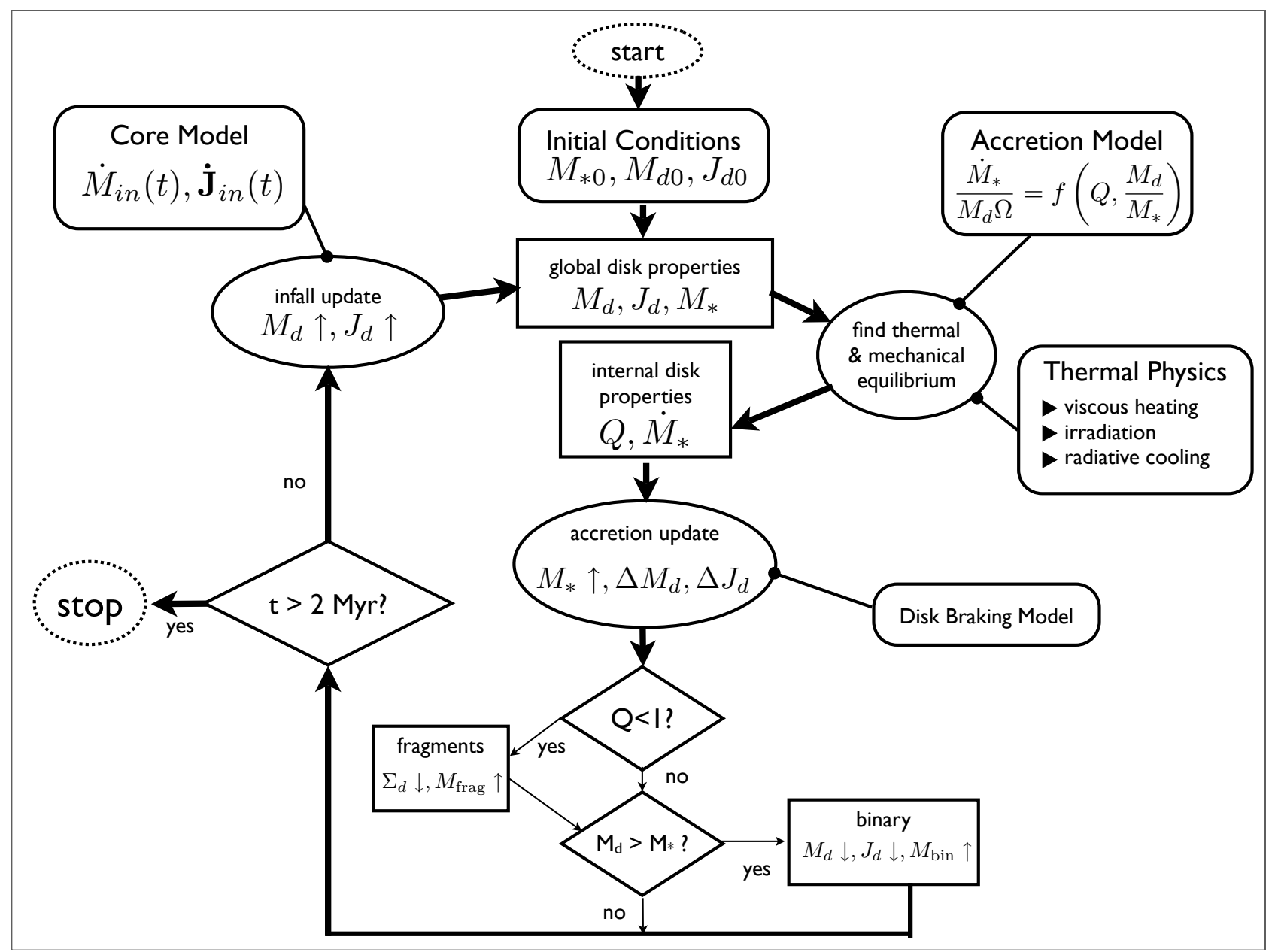

FIG. 3.- A simplified schematic of the decision tree in the code. The primitive variables, $M_{d}, M_{*}$, and $\mathbf{J}_{\mathbf{d}}$, together with the core model, $\dot{M}_{i n}(t)$ and $\dot{\mathbf{J}}_{i n}(t)$, allow for the determination of all disk parameters at each time step. Note that $c_{s}, Q$, and $\dot{M}$ are solved for simultaneously. Once the self-consistent state is found, the values of $Q$ and $\mu$ determine whether either the binary or fragmentation regime has been reached. See 3.7 for a description of the elements in detail.

$\phi_{B} \simeq 2.8$ represents the magnetic enhancement of the turbulent pressure. All together, we predict

$$
\begin{aligned}
\Re_{\text {in }} & =\frac{0.10}{\varepsilon^{2} \phi_{B}^{3 / 2}}\left(\frac{3-k_{\rho}}{1+H_{0}}\right)^{1 / 2} \frac{\left(1-\frac{k_{\rho}}{2}\right)^{1.26}}{\left(k_{\rho}-1\right)^{3 / 2}}\left(1-0.30 k_{\rho}\right) \\
& \rightarrow 0.02\left(\frac{0.5}{\varepsilon}\right)^{2}
\end{aligned}
$$

where the evaluation uses $1+H_{0} \rightarrow 2, \phi_{B} \rightarrow 2.8$, and $k_{\rho} \rightarrow 1.5$.

Importantly, $\Re_{\text {in }}$ is a function of $\left(1+H_{0}\right), \phi_{B}, \epsilon$, and $k_{\rho}$, but not the core mass. We therefore expect similar values of $\Re_{\text {in }}$ to describe all of present-day massive star formation, at least insofar as these other parameters take similar values. Suppose, for instance, that the formation of $10 M_{\odot}$ and $100 M_{\odot}$ stars were both described by the same $\Re_{\text {in }}$. According to equation 27 , the difference in $\mu$ between these two systems would be controlled entirely by the thermal effects that cause them to take different values of $Q$.

A few additional expectations regarding $Q$ itself can be gleaned from the analytical work of Matzner \& Levin (2005) and KM06:
- The Toomre parameter remains higher than unity for low-mass stars $\left(\lesssim 1 M_{\odot}\right)$ in low-column cores $\left(\Sigma_{c, 0} \ll 1\right)$, but falls to unity during accretion for massive stars and for low-mass stars in high-column cores;

- A given disk's $Q$ drops during accretion, reaching unity when the disk extends to radii beyond $\sim 150 \mathrm{AU}$ (in the massive-star case), or to periods larger than $\sim 460 \mathrm{yr}$ (in the case of an optically thick disk accreting from a low-mass, thermal core).

- At the very high accretion rates characteristic of the formation of very massive stars $(\gtrsim 1.7 \times$ $\left.10^{-3} M_{\odot} \mathrm{yr}^{-1}\right)$, disk accretion is strongly destabilized by a sharp, temperature-dependent drop in the Rosseland opacity of dust.

(For more detailed conclusions, see the discussion surrounding equation [35] in (KM06).) With the help of equation (27) we also deduce that more massive stars will have generally higher disk mass fractions, because: (1) they are described (in our model) by the same value of $\Re_{\text {in }}$; (2) more massive stars achieve lower values of $Q$; and (3) in our model, lower $Q$ leads to lower values of 
$\dot{M}_{*} /\left(M_{d} \Omega\right)$, so long as $Q>1.3$. The conclusion that higher-mass stars have relatively more massive disks follows from these three points by virtue of equation 27].

More generally, any effect which causes $\dot{M}_{*} /\left(M_{d} \Omega\right)$ to drop (without affecting $\Re_{\text {in }}$ ) will tend to increase $\mu$, and vice versa; this conclusion is not limited to our adopted disk accretion model.

Within our model, $\dot{M}_{*} /\left(M_{d} \Omega\right)$ increases with $Q$ unless $1<Q<1.3$, in which case the dependence is reversed. Disks ought therefore to traverse from high $Q$ and low $\mu$, to low $Q$ and high $\mu$, until $Q=1.3$; but for $1<$ $Q<1.3, \mu$ and $Q$ should decline together. In physical terms, this reversal represents a flushing of the disk due to the strong angular momentum transport induced by the short wavelength gravitational instability.

We now turn to our suite of numerical models to test these expectations.

\section{RESULTS}

We begin by examining the evolution of disks through their accretion history for a range of stellar masses, determining when and if they are globally or locally unstable, and the dominant mechanism for matter and angular momentum transport through disk lifetimes. Next, we explore how these results are influenced by varying the other main physical parameters: $T_{c}, \Sigma_{c}, \alpha_{\mathrm{MRI}}$, and by varying the angular momentum prescription. For this purpose we first define a fiducial sequence of models in $\S 5.1$ and then expand our discussion to the wider parameter space encompassed by the aforementioned variables. Figures 4-6 show results from our fiducial model, and Figures 8 and 9 explore the effects of our environmental variables.

Because our prescription for disk accretion and fragmentation is necessarily approximate, any specific predictions are unlikely to be accurate. We concentrate instead on drawing useful observational predictions from our models' evolutionary trends.

\subsection{The fiducial model}

Our fiducial model explores a range of masses with a standard set of parameters, which we list in Table 1. For our exploration of the stellar mass parameter space, we allow $\Sigma_{c}$ to vary as $\Sigma_{c}=10^{-1.84}\left(M_{c} / M_{\odot}\right)^{0.75}$ (with an enforced minimum at $0.03 \mathrm{~g} \mathrm{~cm}^{-} 2$ so that $\Sigma_{c}$ varies from $0.03-1 \mathrm{~g} \mathrm{~cm}^{-2}$ across the mass range $\left.0.5-120 M_{\odot}\right)$. This relationship ensures that for our fiducial model, each system is forming at a $\Sigma_{c}$ that is characteristic of observed cores. Enforcing this $\Sigma_{c}-M_{c}$ correspondence specifies the core radius. We explore the effects of $\Sigma_{c}$ independently in 5.3 . All "low mass" runs that are shown e.g. $1 M_{\odot}$, have $\Sigma_{\text {c,low }}=0.03 \mathrm{~g} \mathrm{~cm}^{-2}$, and "high mass", e.g. $15 M_{\odot}$, have $\Sigma_{\mathrm{c}, \text { high }}=0.5 \mathrm{~g} \mathrm{~cm}^{-2}$. All systems start out with an initial stellar mass of $0.10 M_{\odot}$, disk mass of $0.01 M_{\odot}$ and $j_{d}=10^{19}$. Varying these parameters over an order of magnitude effects the initial evolution for a few thousand years, but runs converge quickly. One can find pathological initial conditions, particularly for small mass values. We believe this is due to the lack of sensitivity of a one zone model. The initial disk radius is calculated self-consistently from the amount of mass collapsed into the system at the first time step, the initial $J_{d}$ is typically smaller by a factor of a few than $j_{i n}$.

\begin{tabular}{|c|c|c|}
\hline \hline Parameter & Fiducial & Range \\
\hline$b_{j}$ & 0.5 & $0-1.0$ \\
$\Sigma_{\mathrm{c}, \text { low }}$ & $0.03 \mathrm{~g} \mathrm{~cm}^{-2}$ & $0.03-1 \mathrm{~g} / \mathrm{cm}^{2}$ \\
$\Sigma_{\mathrm{c}, \text { high }}$ & $0.5 \mathrm{~g} \mathrm{~cm}^{-2}$ & $0.03-1 \mathrm{~g} / \mathrm{cm}^{2}$ \\
$T_{c}$ & $20 \mathrm{~K}$ & $10-50 \mathrm{~K}$ \\
$\alpha_{\mathrm{MRI}}$ & 0.01 & $0.001-0.1$ \\
$\phi_{f}$ & 0.05 & $0-0.5$ \\
$\varepsilon$ & 0.5 & N $/ \mathrm{A}$ \\
\hline
\end{tabular}

TABLE 1

FidUCIAL PARAMETERS FOR DISK MODELS FOR LOW AND HIGH MASS STARS, AND THE ACCOMPANYING RANGES EXPLORED.

As illustrated by the evolutionary tracks of accreting stars in the $Q-\mu$ plane in Figure 4 , our model agrees with the general trends of previous work and with the expectations described in $\S 4$, in that low mass systems are stable and have low values of $\mu$, while more massive systems undergo a period of strong gravitational instability (Krumholz et al. 2007b, KM06). Here we see that as we go to higher stellar masses, disks spend more of their time at high $\mu$ and undergoing disk fragmentation. For stars of $\lesssim 1 M_{\odot}, Q$ stays above unity, and the disks remain Toomre stable, although still subject to gravitational instability due to their non-negligable disk masses (see Figure 6). (Note that due to our abrupt shift in the disk irradiation model, there is a small discontinuity in the temperature calculation at the end of accretion which can cause unphysical fragmentation even at low masses, and a jump in $\mathrm{Q}$ at all masses.) The expectation that $Q$ and $\mu$ evolve in opposite directions until $Q<1.3$ is also roughly borne out. However, note that for the $15 M_{\odot}$ star-disk system (right plot), the accretion rate is great enough that there is a build up of mass in the disk once $Q$ reaches unity, and the local instability saturates. This saturation leads to binary formation (see \$5.7).

Figure 5 shows the evolution of $Q$ through the accretion history of a range of masses. We see that disks become increasingly susceptible to fragmentation with increasing mass. Disks born from cores that are smaller than about $2 M_{\odot}$ remain stable against fragmentation throughout their evolution, although we expect moderate spiral structure (as is seen in the models of Lodato \& Rice 2004). Recall that with $\varepsilon=0.5$, a $2 M_{\odot}$ core makes a $1 M_{\odot}$ star-disk system. Figure 6 illustrates the corresponding evolution of $\mu$ throughout the accretion history for the same set of masses. As described in 84 the typical disk mass increases with stellar mass. At high masses, binary formation occurs during the peak of accretion just before $10^{5}$ years, and for stars $\gtrsim 100 M_{\odot}$, there is an early epoch of binary formation at roughly $10^{4}$ years.

We also see that for higher mass cores there are three relatively distinct phases through which disks evolve:

- Type I: Young, < $10^{4}$ yr systems, whose disks are described by small mass fractions and relatively high $Q$. These would appear as early Class 0 sources, deeply embedded in their natal clouds.

- Type II: Systems between $10^{4}-10^{5}$ yrs in age, whose disks are subject to spiral structure, and in high mass systems, fragmentation. Disk mass fractions are $\sim 30 \%-40 \%$, substantially higher than in Type I systems. These disks would appear in Class 0-I sources. 


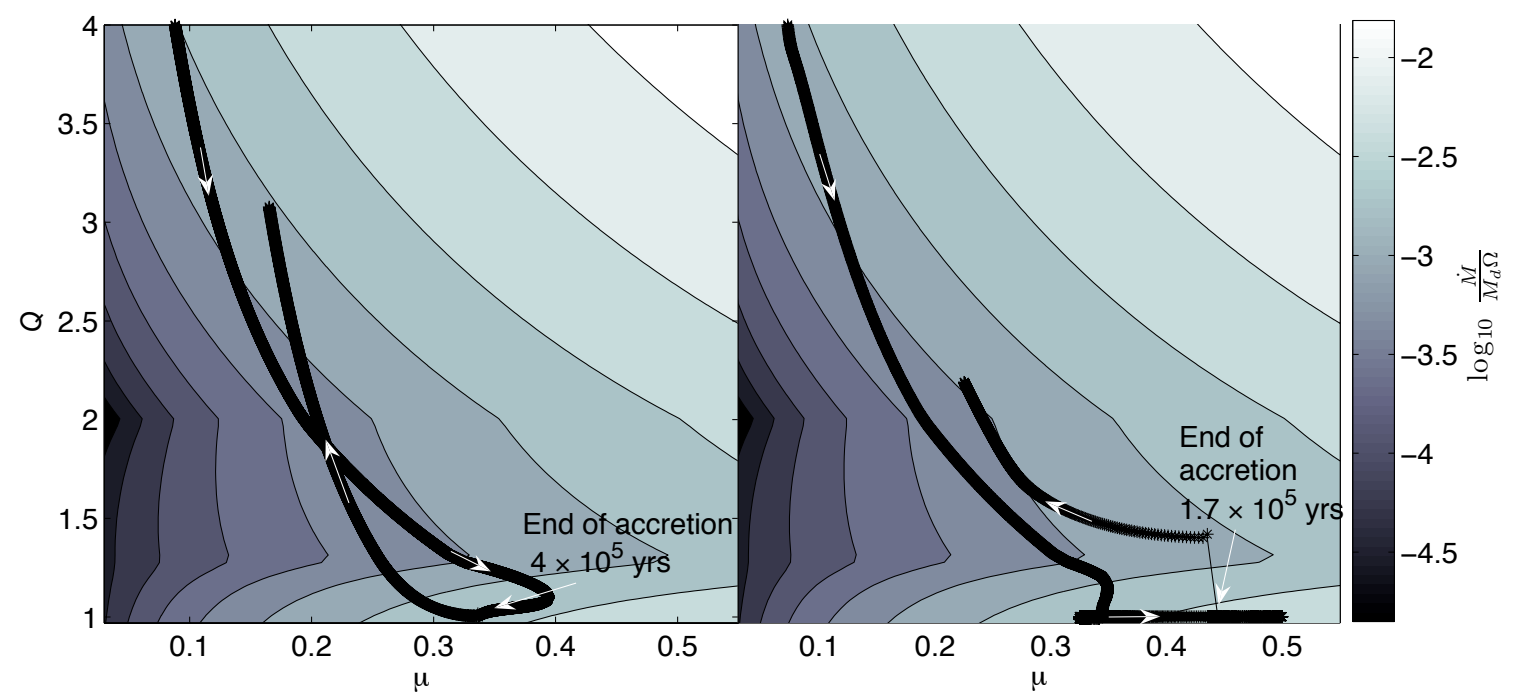

FIG. 4.- Evolutionary tracks in the $Q, \mu$ plane of a $1 M_{\odot}\left(\right.$ left) and $15 M_{\odot}$ (right) final star-disk system overlayed on the contours of our accretion model (contour spacing is identical to Figure 2). The white arrows superposed on the tracks show the direction of evolution in time. The low mass star remains stable against fragmentation throughout its history, while the more massive star undergoes fragmentation and more violent variation in disk mass. The jump a the end of accretion in the $15 M_{\odot}$ system is due to the switch in the irradiation calculation.

- Type III: Systems older than $10^{5}$ yrs, which have stopped accreting from the core, and consequently acquire low disk mass fractions as the disks drain away. These are the disks that are most like those observed in regions of LMSF as Class I objects.

These three stages serve as a useful prediction for future observations; see $\$ 6$ for more details.

\subsection{Influence of vector angular momentum}

The accretion disk's radius plays a critical role in determining whether or not the disk fragments. Consequently, we expect our results to depend somewhat on effects that change the disk's specific angular momentum. Because we track the vector angular momentum of the inner disk, and because our turbulent velocity field is three dimensional, we account for a possible misalignment between the disk's angular momentum axis, $\hat{\mathbf{J}}$, and that of the infalling angular momentum, $\hat{\mathbf{j}}_{\text {in }}$. The wandering and partial cancellation that result provide a more realistic scenario than given by the KM06 analytic approximations, in which vector cancellation is accounted for only in an average sense. In practice, however, the disk and infall remain aligned rather well $\left(\hat{\mathbf{J}} \cdot \hat{\mathbf{j}}_{\text {in }} \sim 0.8\right)$, so misalignment plays only a minor role in limiting the disk size. This is illustrated by Figure 7, in which we compare the disk radius in two numerical realizations of the turbulent velocity field, against one in which $j_{\text {in }}$ has a fixed direction and obeys the KM06 formulae. We also plot the infall circularization radius $R_{\text {circ }}$ (of one numerical realization) for comparison. In general we find that the analytic prescription slightly over-predicts the disk radius at early times; this is partly due to "cosmic" vari-

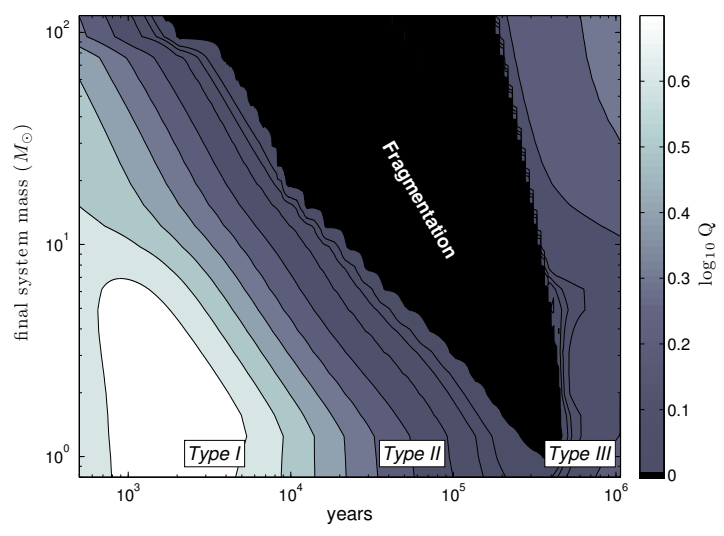

FIG. 5.- Contours of $\mathrm{Q}$ over the accretion history of a range of masses for the fiducial sequence. Masses listed on the y-axis are for the total star-plus-disk system final mass - because the models halt at $2 \mathrm{Myr}$, some mass does remain in the disk. Contours are spaced by 0.3 dex. At low final stellar masses, disks remain stable against the local instability throughout accretion. At higher masses, all undergo a phase of fragmentation. One can see three distinct phases in the evolution as described in 85 Disks start out stable, subsequently develop spiral structure as the disk mass grows and become unstable to fragmentation for sufficiently high masses. As accretion from the core halts, they drain onto the star and once again become stable.

ance in the numerical realization, and partly due to diskinfall misalignment.

\subsection{Varying $\Sigma_{c}$}

We explore the effect of individual parameters by considering one or two systems along our fiducial sequence, and varying parameters one by one relative to their fidu- 


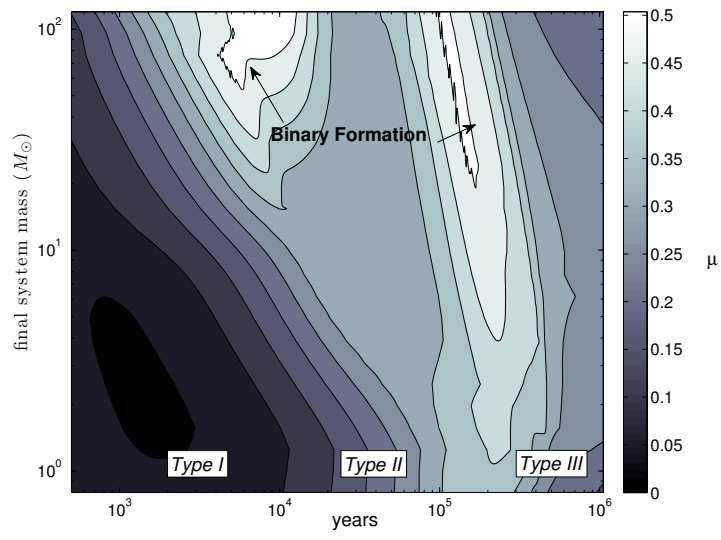

FIG. 6.- Contours showing the evolution of $\mu=\frac{M_{d}}{M_{d}+M_{*}}$ for the fiducial sequence. Each contour shows an increase of 0.05 in $\mu$. Again one can see the division into three regimes: low mass disks at early times, higher mass, unstable disks that may form binaries during peak accretion times, and low mass disks that drain following the cessation of infall. Systems destined to accrete up to $\sim 70 M_{\odot}$ or more experience two epochs of binary formation in our model. In these systems the accretion from the core exceeds the maximum disk accretion rate very early, causing the disk mass to build up quickly.

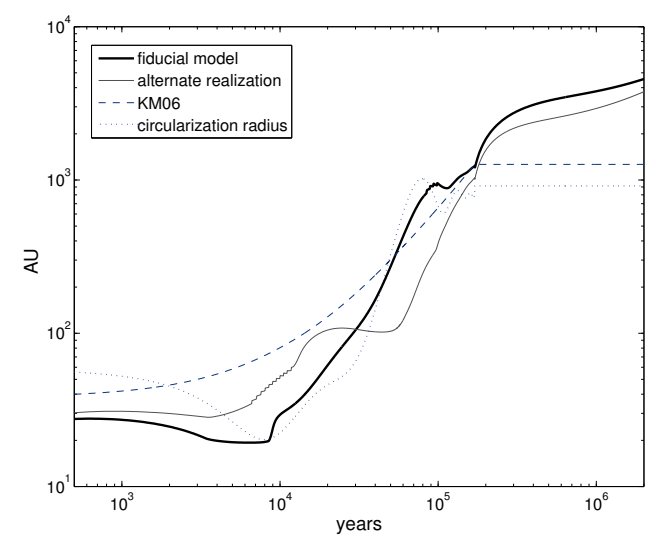

FIG. 7.- Comparison of disk radius over the evolution of a 20 $M_{\odot}$ star-disk system in four cases: the KM06 analytic calculation, the circularization radius of the currently accreting material, and two realizations of the numerical model. The analytic case overestimates the expected radius at early times because it does not allow for cancellation of vector angular momentum. Similarly the circularization radius is an overestimate because the disk has no "memory" of differently oriented j. At later times, the circularization radius approaches the standard radius calculation for that realization (thick black line) demonstrating the concentration of turbulent power at large scales.

cial values. First, we vary $\Sigma_{c}$ over $0.03-1 \mathrm{~g} \mathrm{~cm}^{-3}$, spanning the range from isolated to intensely clustered star formation (Plume et al. 1997). Column density affects star formation in two primary ways: it influences the core radius (by determining the confining pressure) and the accretion rate during collapse (again, by setting the outer pressure and thus the velocity dispersion). However, these two effects counteract one another: smaller values of $\Sigma_{c}$ correspond to larger cores and larger, thus more unstable disks $\left(R_{d} \propto \Sigma_{c}^{-1 / 2}\right)$, but smaller $\Sigma_{c}$ also leads to lower accretion rates and thus stabler disks $\left(\dot{M} \propto \Sigma_{c}^{3 / 4}\right)$. The thermal balance of the disk midplane is affected by these trends. An analysis by KM06 (see their equation [35]) shows that higher $\Sigma_{c}$ inhibits fragmentation if the disk temperature is dominated by viscous heating (which is proportional to the accretion rate), but enhances fragmentation if irradiation dominates (when the increase in accretion generated heating is insignificant), and that the two effects are comparable along our fiducial model sequence. We therefore expect fragmentation to be quite insensitive to $\Sigma_{c}$, for massive star formation along our fiducial sequence. This is precisely what we find in our models: disks born from lower- $\Sigma_{c}$ cores, in lower pressure environments, evolve in essentially the same way, but more slowly.

In contrast, disks around low-mass stars - those with final masses comparable to the thermal Jeans mass are stable at low $\Sigma_{c}$ (as predicted by Matzner \& Levin 2005 ), and because irradiation dominates at larger radii, higher $\Sigma_{c}$ tend to enhance fragmentation there. Figure 8 illustrates the evolution of $Q$ for a $1 M_{\odot}$ accreting star for a range of column densities.

\subsection{Varying $T_{c}$}

Observations of infrared dark clouds, and sub-mm core detections find typical temperatures from $10-50 \mathrm{~K}$ (Johnstone et al. 2001). In our models $T_{c}$ determines the amount of thermal, and therefore turbulent, support: higher temperatures require less turbulent support in the core. Temperature also sets the thermal Jeans mass $M_{\mathrm{th}}$ within the McKee \& Tan (2003) two component core model. Accretion from this thermal region leads to more stable disks; therefore, higher core temperatures increase the mass of a star which can accrete stably.

Figure 8 shows the evolution of $Q$ during the accretion of a system with final mass $1 M_{\odot}$ over a range of temperatures (all other parameters take their fiducial values). The difference in evolution is negligible for high mass stars, as these accrete from supersonic cores.

\subsection{Varying $\alpha_{\mathrm{MRI}}$}

This work is not an exploration of the detailed behavior of the MRI; we include it as the standard mechanism for accretion in the absence of gravitational instabilities, which in most scenarios (aside perhaps from low mass stars whose disks Shu et al. 2007 have argued may be strongly sub-Keplerian) overpower the MRI. However, the strength of the MRI does influence the transition to gravitationally dominated accretion in the $Q-\mu$ plane as shown in Figure 2. The strength of the MRI also influences the maximum disk mass obtained before gravitational instabilities set in: higher values of $\alpha_{\mathrm{MRI}}$ reduce the influence of gravitational instabilities by insuring that the disk drains more quickly, whereas lower values expedite the transition to gravitational instability driven accretion. Figure 9 shows the influence of $\alpha_{\mathrm{MRI}}$ on $\mu$; the influence on $Q$ is less dramatic: the descent of $Q$ towards unity is marginally delayed for the strong MRI case.

\subsection{Varying $b_{j}$}

Our most uncertain variable is the braking index $b_{j}$, which determines the rate of angular momentum exchange with an outer disk. However, disk evolution turns out to be rather insensitive to this parameter. The primary reason for this is the concentration of power in the 

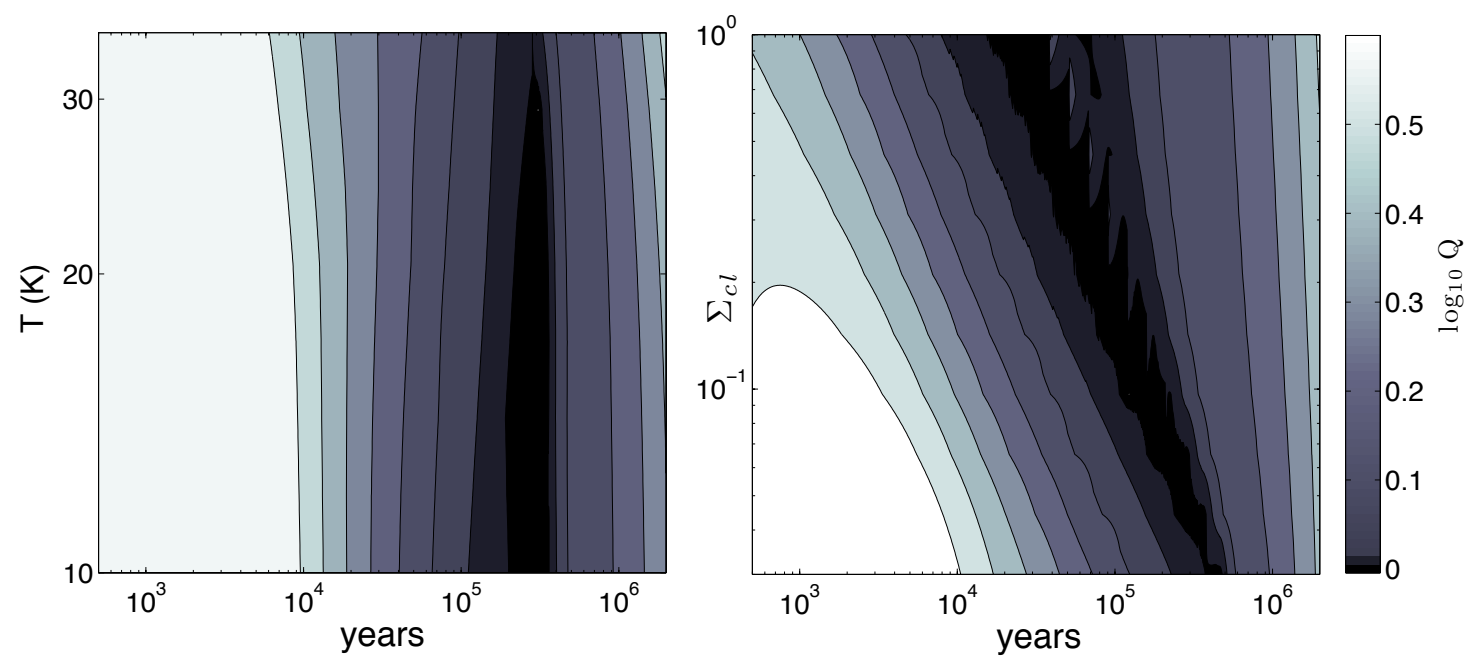

FIG. 8. - Contours of Q showing the effect of initial core temperature $T_{c}$ (left) and $\Sigma_{c}$ (right) on the evolution of a $1 M_{\odot}$ final star-disk system. Contour spacing is $0.1 \mathrm{dex}$ (except the lowest two contours which are spaced by .05 dex). Increasing $\Sigma_{c}$ tends to marginally destabilize the disk, while higher temperatures stabilize the disk. We exclude temperatures too high for the $2 M_{\odot}$ core to collapse given its initial density, i.e., those above $40 \mathrm{~K}$.

turbulent velocity field on the largest scales: $j_{\text {in }}$ is always large compared to the disk-average $j$. This reduces the importance of the loss term in equation $(22)$. As a result, although the period in which the disk is fragmenting is reduced in the high $b_{j}$ case, it is merely postponed by $\sim 10^{4}$ yrs. The braking index does have moderate influence on the disk mass during the peak of accretion, and thus on the formation of binaries. Figure 9 shows the evolution of $\mu$ for a system accreting towards $15 M_{\odot}$ from a $30 M_{\odot}$ core. Here one can see the influence of $b_{j}$ on binary formation. Low values of $b_{j}$ corresponding to higher net angular momentum produce binaries at lower masses by allowing the disk mass to grow larger. Notably, even for the $15 M_{\odot}$ final mass star shown here, the smallest mass for which binaries form in the fiducial model, the change in disk mass is only $\sim 10 \%$.

\subsection{The formation of binaries}

Within the context of our model for disk fission into a binary system, (described in \$3.6), the formation of a companion is strongly dependent on the infalling angular momentum distribution, and on the turbulent velocity profile of the particular core. In our fiducial model, binary formation occurs for cores above $30 M_{\odot}$. For cores $\gtrsim 140 M_{\odot}$, there are two epochs of binary formation, the first one at roughly $10^{4}$ years. This mass boundary is quite sensitive to our conservative threshold for disk fission, $\mu=0.5$ : binaries may well form at lower values of $\mu$, and thus at lower masses (see Figure 6). The mass of the binaries that form increases with initial core mass. This increase simply indicates that the mass ratio exceeds the critical value for more time, as we do not include a mechanism for accretion onto the binary. As such, we do not predict values for the binary mass ratio $q$, but simply indicate the regimes in which binary formation seems likely. The $30 M_{\odot}$ core cut-off is fairly robust to variations in $\Sigma_{c}, T_{\mathrm{c}}$ and $b_{j}$ over the ranges discussed above for our fiducial turbulent field. Cosmic variance in the field from one realization to another has a much larger effect on binary formation than any of our other model parameters (aside from $\mu_{\text {crit }}$ ).

In our fiducial model disk fission only occurs when the gravitational instability has saturated and $Q \sim 1$. This means that the disk is draining at the maximum rate given its mass. If matter is falling in from the core more rapidly than this rate, the disk mass will increase: if the accretion rate from the core exceeds the maximum rate at $Q=1$ and $\mu=0.50$, disk fission occurs. In our fiducial model, this corresponds to an accretion rate on to the disk: $\dot{M}_{\mathrm{in}} / M_{d} \Omega=10^{-2.36}$. The early epoch of binary formation at very high masses is a consequence of this limit: since the accretion rates begin to exceed the critical rate sooner, the disk's mass increases earlier in its evolution. This critical value is in agreement with the prediction of KM06 that disks are sharply destabilized when accreting at rates higher than $1.7 \times 10^{-3} M_{\odot} \mathrm{yr}^{-1}$.

The time at which binaries form is also very dependent on the angular momentum profile. In the fiducial model, the lowest mass binaries form during the peak of accretion, at $\sim 10^{5}$ years, but as the final system mass increases, binary formation pushes to earlier times $\sim 10^{4}$ years. In certain runs we find earlier binary formation at smaller masses $\left(<10^{4}\right.$ years $)$ when there is a peak in the infalling angular momentum profile which rapidly sends $Q$ towards unity. The presence of binaries in much of our parameter space illustrates that heavy circumbinary disks may be critical to binary evolution.

Observations suggest that a range of binary systems exist as a result of variations in angular momentum as evidenced by the presence or lack of disks around each component. Submillimeter observations of lower mass objects in Taurus have revealed evidence for a binary with circumstellar and circumbinary disks (Osorio et al. 2003), where the binaries are close enough to cause disk 

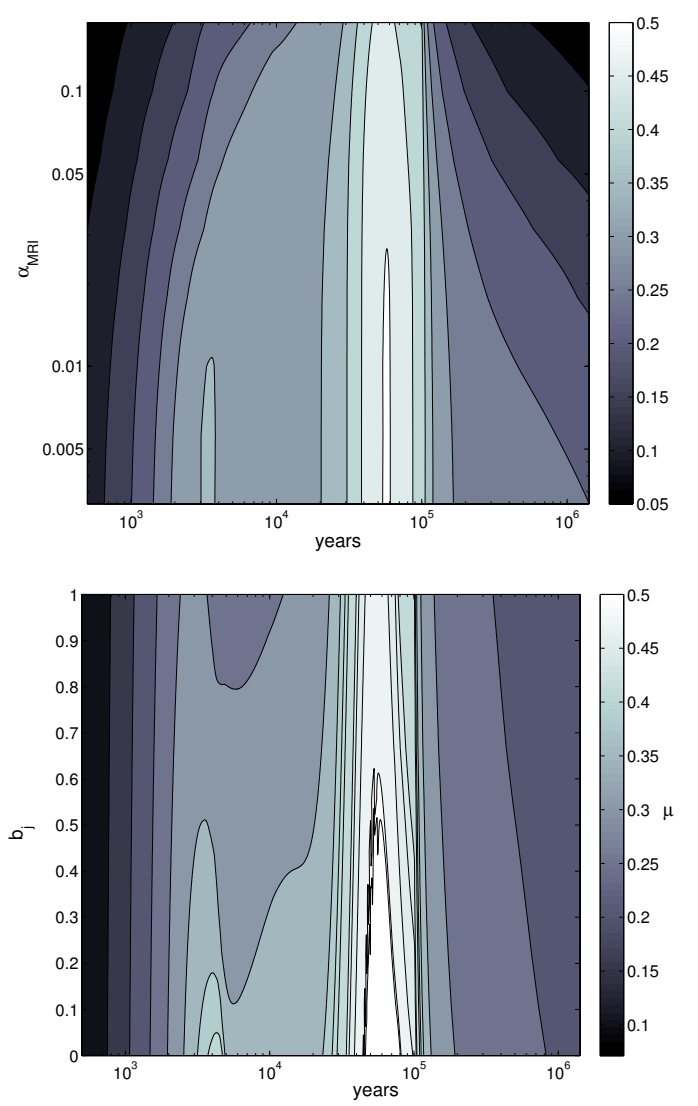

FIG. 9.- Contours in $\mu$ illustrating the influence of varying $\alpha_{\mathrm{MRI}}$ (top) and the braking index, $b_{j}$ (bottom) for a star-disk system of final mass $15 M_{\odot}$, the lowest mass at which a binary forms in our fiducial model. Contours of $\mu$ are spaced by 0.05 . The upper plot shows the effect of varying $\alpha_{\mathrm{MRI}}$ from $10^{-2.5}-10^{-1.5}$. While the change has little effect on the evolution of $Q$, the disk fraction $\mu$ decreases with increasing $\alpha_{\mathrm{MRI}}$. As a result, the mass at which binary formation begins is pushed to higher masses. The lower plot shows the effect of varying the braking index $b_{j}$. An increase of $b_{j}$ lowers the disk angular momentum, reducing the disk mass and inhibiting binary formation. Note that the variation in disk mass is only $\sim 10 \%$.

truncation ( 45 AU). Anglada et al. (2004) have found another Class 0/I binary system in NGC 1333 in which only the primary has a disk: the diversity of systems is likely due to the variations in angular momentum of the infalling material. As Bate \& Bonnell (1997) suggest, binaries forming from low angular momentum material will likely not form their own disks, while those with higher angular momentum may. It seems plausible that the absence or presence of secondary disks is indicative of the formation process of the system.

As illustrated by these observations, the dependence we find on core angular momentum is a sensible outcome: one expects the chance rotation to have a stronger effect on multiplicity than other parameters like temperature and density, which set the minimum fragmentation mass. We emphasize that we are only exploring one possible path for binary formation, and predict that disk fragmentation is an important, if not the dominant mechanism at high masses and column densities. This is especially true since, as argued by Krumholz (2006), who has shown that for massive stars, once the central core has turned on, the Jeans mass rapidly increases due to the stellar luminosity, significantly reducing the possibility for Jeans-instability induced core fragmentation.

\section{OBSERVABLE PREDICTIONS}

Our models make strong predictions for the masses and morphologies of disks during the embedded, accreting phase, and these will be directly testable with future observations. Detailed calculations based on radiationhydrodynamic simulations of massive protostellar disks indicate that disks with $\mu$ of a few tenths around stars with masses $\gtrsim 8 M_{\odot}$, corresponding to embedded sources with bolometric luminosities $\gtrsim 10^{4} L_{\odot}$, should produce levels of molecular line emission that are detectable and resolvable with ALMA in the sub-millimeter out to distances of a few kpc, and with the EVLA at centimeter wavelengths at distances up to $\sim 0.5 \mathrm{kpc}$ (Krumholz et al. 2007a). The ALMA observations will be particularly efficient at observing protostellar disks, since ALMA's large collecting area will enable it to map a massive disk at high resolution in a matter of hours. Dust continuum emission at similar wavelengths should be detectable at considerably larger distances, although the lack of kinematic information associated with such observations makes interpretation more complex. Regardless of whether dust or lines are used, observations using ALMA should be able to observe a sample of hundreds of protostellar disks around embedded, still-accreting sources, with masses up to several tens of $M_{\odot}$.

The main observational prediction of our model is the existence of type II disks - those with $\mu$ of a few tenths or greater and $Q \approx 1-$ and the mass and time-dependence of the type II phase. Examining Figures 5 and 6 , we see that our model predicts that protostellar cores with masses $\lesssim 2 M_{\odot}$ should experience only a very short type II disk phase, or none at all. In contrast, cores with larger masses have type II disks for a fraction of their total evolutionary time that gets larger and larger as the core mass rises, reaching the point where type II disks are present during essentially the entire class 0 , accreting phase for cores $\gtrsim 100 M_{\odot}$ in mass.

Type II disks have several distinct features that should allow observations to distinguish them from type I or type III disks, and from older disks like those around $\mathrm{T}$ Tauri and Herbig AE stars. First, since type II disks are subject to strong gravitational instability, they should have strong spiral arms, with most of the power in the $m=1$ or $m=2$ modes. This is perhaps the easiest feature to pick out in surveys, since it simply requires observing the disk morphology and can therefore be measured using continuum rather than lines.

Second, because their self-gravity is significant, type II disks will deviate from Keplerian rotation due to nonaxisymmetric motions, and will also be super-Keplerian in their outer parts compared to their inner ones. The latter effect arises because, when the disk mass is comparable to the stellar mass, the enclosed mass rises as one moves outward in the disk. Recent work by Torrelles et al. (2007) provides a possible example of this phenomenon. The source HW2 in Cepheus A is predicted to have a central mass of order $15 M_{\odot}$, and a disk radius of 300AU, with a temperature slightly under $200 \mathrm{~K}$ (Patel et al. 2005: Torrelles et al. 2007). High resolution VLA observations now show evidence of non-Keplerian rotation (Jiménez-Serra et al. 2007), consistent with our 
predictions for type II disks.

Third, a type II disk is massive enough for the stardisk system center of mass to be significantly outside of the stellar surface if the disk possesses significant nonaxisymmetry. As a result, the star will orbit the center of mass of the system, and this will produce a velocity offset of a few $\mathrm{km} \mathrm{s}^{-1}$ between the stellar velocity and the zero velocity of the inner, Keplerian parts of the disk (Bertin \& Lodato 1999, Rice et al. 2003, Krumholz et al. 2007a). This should be detectable if the stellar velocity can be measured, which may be possible using Doppler shifts of radio recombination lines for stars producing hypercompact HII regions, or using proper motions for stars with large non-thermal radio emission (Bower et al. 2003). In fact recent work by Torrelles et al. (2007) has observed said offset. As suggested by Lodato \& Bertin (2001, 2003), one could also look for the effect in the unresolved radio emission from FU Orionis objects.

A final point concerns the limited range of the disk-tosystem mass ratio in our simulations, with $0.2<\mu<0.5$ during most of embedded accretion (our type II disks). The upper envelope of $\mu$ depends in part on our binary fragmentation threshold $\mu=0.5$. However, in the absence of disk fission, disks in our fiducial model never grow larger than $\mu=0.55$. The fact that most accretion occurs with $\mu \sim 0.3$ provides strong evidence that accretion disks do not become very massive compared to the central point mass (as argued by Adams et al. 1989). Current observations such as those of Cesaroni (2005) describe massive tori with sub-Keplerian rotation and comparable infall and rotation velocities. These structures are distinct from the disks that we model: our finding that disks hover around $\mu=0.3$ suggests that higher resolution observations may reveal the Keplerian structures within the tori. The underlying physical reason for this is that it is not possible to support a mass comparable to the central star in a rotationally supported disk for long periods of time; gravitational instabilities will destabilize such a disk on orbital timescales, causing it to lose mass either through rapid accretion or fragmentation.

\section{CONCLUSIONS}

We have constructed a simple, semi-analytic one-zone model to map out the parameter space of disks in $Q-\mu$ space across a range of stellar masses throughout the Class 0 and Class I stage, pushing into the Class II phase. We include angular momentum transport driven by two different mechanisms: gravitational instability and MRI transport modeled by a constant $\alpha$. Our model for angular momentum infall is unique in that we keep track of an inner and outer disk, and infall direction so that cancellation may occur as the infall vector rotates. We allow for heating by the central star, viscous dissipation and a background heat bath from the cloud accounting for both the optically thin and optically thick limit within the disk and accreting envelope. By requiring that the disk maintain mechanical and thermal equilibrium, we determine the midplane temperature at each time step, and thus $Q$ in the disk.

\subsection{Caveats}

In interpreting the results of our calculations, it is important to keep several caveats in mind. Our model for fragmentation, though rooted in simulations, includes one important assumption: no matter how violently unstable a disk becomes, it can always fragment, return to a marginally stable state, and continue accreting. While the existence of stars well into the mass regime of fragmentation makes this outcome seem likely, it has yet to be demonstrated in simulations. Equally untested is the hypothesis that when fragmentation is strong enough, i.e., when $\dot{M}_{\text {in }} \gg c_{s}^{3} / G$ so that $Q \ll 1$ (Gammie 2001), accretion onto the central star will be choked off. KM06 have argued that accretion is sharply destabilized when its rate exceeds $1.7 \times 10^{-3} M_{\odot} \mathrm{yr}^{-1}$, due to a drop in the Rosseland opacity, and that this may be related to the stellar upper mass limit.

In order to explore a wide parameter space, we do not carry out detailed hydrodynamic calculations to determine the onset of instability, but instead use results from previous simulations, and develop analytic formulae that describe behavior intermediate between the regimes which they explore. Although our approach is very approximate, it can be made increasingly more realistic as additional numerical simulations become available. Due to our one-zone prescription, we cannot resolve spiral structure or measure the degree of non-Keplerian motion. In addition, we do not follow the evolution of fragments, nor their interaction with the disk. Although we allow for the formation of binaries, we do not follow their evolution and accretion, which limits our ability to make predictions about mass ratios and angular momentum transfer between the disk and the companion. Our model for angular momentum infall is responsible for the largest uncertainty in our conclusions because different realizations of the turbulent velocity field can alter the disk size at a given epoch by a factor of a few. Nevertheless, these variations are well within the analytic expectations for range of angular momenta in cores (KM06). Moreover, our approach aims only to predict characteristics of the outer accretion disk, and lacks the resolution to track the radial profiles of the disk's properties.

Lastly, recall that our models rely on the fundamental assumption ( $\S 3.1$ that a disk's behavior can be separated into dynamical and thermal properties, and in particular that its dynamics are governed primarily by its mass fraction $\mu$ and Toomre parameter $Q$.

With these caveats in mind, we summarize our results for two different regimes: $<2 M_{\odot}$ and $>2 M_{\odot}$.

\subsection{The Low Mass Regime}

Our fiducial models predict that low mass stars will have higher values of $\mu$ than typically assumed during early phases of formation. However, they should remain stable against fragmentation throughout their evolution, dominated by MRI, long wavelength gravitational instability, and once again MRI through their evolution through the three types of disks discussed in \$5. During the main accretion phase, disks will have masses of order $30 \%$ of the system mass. Typical outer radii are of order $50 \mathrm{AU}$, with outer temperatures of $40 \mathrm{~K}$ during the main accretion phase, dropping to $\sim 10 \mathrm{~K}$ at 2 Myr. The surface density is $10-20 \mathrm{~g} \mathrm{~cm}^{-2}$ during the main accretion phase, dropping off rapidly at late times causing the disk to become optically thin to its own radiation. As accretion shuts down, and disks grow due to conservation of angular momentum, two key effects must 
be considered: truncation and heating by other stars. At distances of $1000 \mathrm{AU}$, very tenuous disks are prone to truncation by passing stars particularly in denser clusters where average stellar densities are as high as $10^{5}$ stars $\mathrm{pc}^{-3}$ (Hillenbrand \& Hartmann 1998). Similarly, as the disk edge extends towards other, potentially more luminous stars, the actual flux received will increase, heating the disk above the $\sim 10 \mathrm{~K}$ temperature that we routinely find (Adams et al. 2006).

For core column densities more typical of high-mass star forming regions, local instabilities do set in, despite the stabilizing influence higher temperatures associated with these regions (neglecting the effects of nearby stars). This implies that environment may be important in understanding disk evolution.

In contrast to our previous work (Kratter \& Matzner 2006 Matzner \& Levin 2005), we find tragmentation at smaller radii. This is primarily due to our modified model for $\alpha_{\mathrm{GI}}$, which predicts lower accretion rates and consequently more fragmentation then previously assumed. We note that our results for low-mass systems (final mass $\sim 1 M_{\odot}$ ) are rather sensitive to details of the model, such as the value of $\alpha_{\mathrm{MRI}}$ and the way it is combined with $\alpha_{\mathrm{GI}}$.

\subsection{The High Mass Regime}

For more massive stars, we find high values of $\mu \sim 0.35$ and an extended period of local fragmentation as the accretion rates peak. Temperatures at the disk outer edge at $\sim 200 \mathrm{AU}$ approach $100 \mathrm{~K}$ for systems $>15 M_{\odot}$ during accretion. surface densities hover around $50 \mathrm{~g} \mathrm{~cm}^{-2}$ during the main accretion phase, although by $2 \mathrm{Myr}$, the disks become optically thin in the FIR, as expected. Binary formation occurs regularly for cores of order $30 M_{\odot}$ and higher, though as discussed in 3.6 this is strongly dependent on the cosmic variance of the angular momentum: cores as small as $20 M_{\odot}$ form binaries in our model when there is excess angular momentum infall. Although fragments accrete with the disk according to equation 25], more massive stars maintain a small mass in fragments $\left(10^{-1}-10^{-2} M_{\odot}\right)$ in the disk when we end our simulations, suggesting that fragments may persist to form low mass companions, as predicted in KM06 and suggested by the simulations of Krumholz et al. (2007b).

Unlike their low mass counterparts, the conclusions we draw for massive stars are minimally effected by the environmental variables in our model. For the entire range of temperatures, densities, and nearly all angular momentum realizations, the conclusions listed above hold true.

The authors are grateful to an anonymous referee for insightful comments that improved and clarified this work. The authors would also like to thank Norm Murray, Debra Shepherd, Guiseppe Lodato, and W. Ken Rice for helpful discussions. KMK is supported by a U. Toronto fellowship. CDM's research is funded by NSERC and the Canada Research Chairs program. MRK is supported through Hubble Fellowship grant \#HSF-HF01186 awarded by the Space Telescope Science Institute, which is operated by the Association of Universities for Research in Astronomy, Inc., for NASA, under contract NAS 5-26555. This research was supported in part by the National Science Foundation under Grant No. PHY0551164 .

\section{REFERENCES}

Adams, F. C., Proszkow, E. M., Fatuzzo, M., \& Myers, P. C. 2006, ApJ, 641, 504

Adams, F. C., Ruden, S. P., \& Shu, F. H. 1989, ApJ, 347, 959

Anglada, G., Rodríguez, L. F., Osorio, M., Torrelles, J. M., Estalella, R., Beltrán, M. T., \& Ho, P. T. P. 2004, ApJ, 605, L137

Balbus, S. A., \& Papaloizou, J. C. B. 1999, ApJ, 521, 650

Bate, M. R., \& Bonnell, I. A. 1997, MNRAS, 285, 33

Bertin, G., \& Lodato, G. 1999, A\&A, 350, 694

Beuther, H. 2006, in IAU Symposium 237: Triggered Star Formation in a Turbulent ISM, ed. B. G. Elmegreen \& J. Palous, in press, astro-ph/0610411

Beuther, H., Churchwell, E. B., McKee, C. F., \& Tan, J. C. 2007, in Protostars and Planets V, B. Reipurth, D. Jewitt, and K. Keil (eds.), University of Arizona Press, Tucson, 951 pp., 2007. p.165-180, ed. B. Reipurth, D. Jewitt, \& K. Keil, 165-180

Boley, A. C., Mejía, A. C., Durisen, R. H., Cai, K., Pickett, M. K., \& D'Alessio, P. 2006, ApJ, 651, 517

Bonnell, I. A., Vine, S. G., \& Bate, M. R. 2004, MNRAS, 349, 735

Bower, G. C., Plambeck, R. L., Bolatto, A., McCrady, N., Graham, J. R., de Pater, I., Liu, M. C., \& Baganoff, F. K. 2003, ApJ, 598, 1140

Burkert, A., \& Bodenheimer, P. 2000, ApJ, 543, 822

Cai, K., Durisen, R. H., Boley, A. C., Pickett, M. K., \& Mejia, A. C. 2007 , ArXiv e-prints, 706

Caselli, P., \& Myers, P. C. 1995, ApJ, 446, 665

Cesaroni, R. 2005, Ap\&SS, 295, 5

Cesaroni, R., Galli, D., Lodato, G., Walmsley, C. M., \& Zhang, Q. 2007, in Protostars and Planets V, B. Reipurth, D. Jewitt, and K. Keil (eds.), University of Arizona Press, Tucson, 951 pp., 2007., p.197-212, ed. B. Reipurth, D. Jewitt, \& K. Keil, 197-212

Cesaroni, R., Galli, D., Lodato, G., Walmsley, M., \& Zhang, Q. 2006, Nature, 444, 703
Chiang, E. I., \& Goldreich, P. 1997, ApJ, 490, 368

Dubinski, J., Narayan, R., \& Phillips, T. G. 1995, ApJ, 448, 226

Fisher, R. T. 2004, ApJ, 600, 769

Gammie, C. F. 2001, ApJ, 553, 174

Goodman, A. A., Benson, P. J., Fuller, G. A., \& Myers, P. C. 1993, ApJ, 406, 528

Goodwin, S. P., Whitworth, A. P., \& Ward-Thompson, D. 2004, A\&A, 414, 633

Griv, E. 2006, MNRAS, 365, 1007

Hillenbrand, L. A., \& Hartmann, L. W. 1998, ApJ, 492, 540

Hueso, R., \& Guillot, T. 2005, A\&A, 442, 703

Jayawardhana, R., Coffey, J., Scholz, A., Brandeker, A., \& van Kerkwijk, M. H. 2006, ApJ, 648, 1206

Jiménez-Serra, I., Martín-Pintado, J., Rodríguez-Franco, A., Chandler, C., Comito, C., \& Schilke, P. 2007, ApJ, 661, L187

Johnstone, D., Fich, M., Mitchell, G. F., \& Moriarty-Schieven, G. 2001, ApJ, 559, 307

Keto, E. 2007, ApJ, 666, 976

Kratter, K. M., \& Matzner, C. D. 2006, MNRAS, 373, 1563

Krumholz, M. R. 2006, ApJ, 641, L45

Krumholz, M. R., Klein, R. I., \& McKee, C. F. 2007a, ApJ, 665, 478

—. 2007b, ApJ, 656, 959

Krumholz, M. R., \& Thompson, T. A. 2007, ApJ, 661, 1034

Larson, R. B. 1984, MNRAS, 206, 197

Laughlin, G., \& Rozyczka, M. 1996, ApJ, 456, 279

Li, Z.-Y., \& Shu, F. H. 1996, ApJ, 472, 211

Lin, D. N. C., \& Pringle, J. E. 1987, MNRAS, 225, 607

—. 1990, ApJ, 358, 515

Lodato, G., \& Bertin, G. 2001, A\&A, 375, 455

-. 2003, A\&A, 408, 1015

Lodato, G., \& Rice, W. K. M. 2004, MNRAS, 351, 630

-. 2005, MNRAS, 358, 1489

Matzner, C. D. 2007, ApJ, 659, 1394 
Matzner, C. D., \& Levin, Y. 2005, ApJ, 628, 817

Matzner, C. D., \& McKee, C. F. 2000, ApJ, 545, 364

McKee, C. F., \& Tan, J. C. 2002, Nature, 416, 59

-. 2003, ApJ, 585, 850

McLaughlin, D. E., \& Pudritz, R. E. 1997, ApJ, 476, 750

Myers, P. C., \& Fuller, G. A. 1992, ApJ, 396, 631

Nakamoto, T., \& Nakagawa, Y. 1994, ApJ, 421, 640

-. 1995, ApJ, 445, 330

Nakamura, F., \& Li, Z.-Y. 2007, Ap.J, 662, 395

Osorio, M., D'Alessio, P., Muzerolle, J., Calvet, N., \& Hartmann, L. 2003, ApJ, 586, 1148

Patel, N. A., Curiel, S., Sridharan, T. K., Zhang, Q., Hunter, T. R., Ho, P. T. P., Torrelles, J. M., Moran, J. M., Gómez, J. F., \& Anglada, G. 2005, Nature, 437, 109

Pessah, M. E., Chan, C.-k., \& Psaltis, D. 2007, ArXiv e-prints, 705

Plume, R., Jaffe, D. T., Evans, N. J., I., Martin-Pintado, J., \& Gomez-Gonzalez, J. 1997, ApJ, 476, 730

Porter, D. H., Pouquet, A., \& Woodward, P. R. 1992, Physical Review Letters, 68, 3156
Rafikov, R. R. 2001, MNRAS, 323, 445

Rice, W. K. M., Armitage, P. J., Bate, M. R., \& Bonnell, I. A. 2003, MNRAS, 339, 1025

Rodríguez, L. F., Garay, G., Brooks, K. J., \& Mardones, D. 2005, ApJ, 626, 953

Sellwood, J. A., \& Carlberg, R. G. 1984, ApJ, 282, 61

Semenov, D., Henning, T., Helling, C., Ilgner, M., \& Sedlmayr, E. 2003, A\&A, 410, 611

Shakura, N. I., \& Sunyaev, R. A. 1973, A\&A, 24, 337

Shu, F. H. 1977, ApJ, 214, 488

Shu, F. H., Galli, D., Lizano, S., Glassgold, A. E., \& Diamond, P. H. 2007, ArXiv e-prints, 705

Toomre, A. 1964, ApJ, 139, 1217

Torrelles, J. M., Patel, N. A., Curiel, S., Ho, P. T. P., Garay, G., \& Rodríguez, L. F. 2007, ApJ, 666, L37

Vorobyov, E. I., \& Basu, S. 2005, ApJ, 633, L137

-. 2006, ApJ, 650, 956 